\title{
Delay, state, and parameter estimation in chaotic and hyperchaotic delayed systems with uncertainty and time-varying delay
}

\author{
Shahab Torkamani • Eric A. Butcher
}

Received: 16 April 2013 / Revised: 20 May 2013 / Accepted: 22 May 2013 / Published online: 14 June 2013

(C) Springer-Verlag Berlin Heidelberg 2013

\begin{abstract}
A novel approach is proposed for simultaneous estimation of states, delay and parameters of nonlinear chaotic and hyperchaotic delayed systems with constant delay as well as simultaneous estimation of states and parameters for such delayed systems with time-varying delay. The approach exploits continuous time approximation and stochastic optimal filtering. Also, an innovative technique is proposed to approximately compute the Lyapunov exponents of a nonlinear delayed system in order to determine the parameter values for which the system becomes chaotic or hyperchaotic. The model used in this approach contains two different source of considerable uncertainty. The approach is successfully implemented for state, parameter and delay estimation on various forms of time delayed Lorenz system and delayed Hopfield neural network including chaotic and hyperchaotic cases with constant and time-varying delays. In case of delayed Hopfield neural network, the performance of the approach is shown to be superior compared with two other existing approaches.
\end{abstract}

Keywords Stochastic estimation - Chaotic delayed oscillators · Hyperchaotic delayed oscillators · Uncertainty · Time delay systems · Delay estimation

\section{Introduction}

Since Mackey and Glass in 1977 [1] found chaotic behavior in a delay differential equation (DDE) model of blood pro-

S. Torkamani $(\varangle) \cdot$ E. A. Butcher

Department of Mechanical and Aerospace Engineering, New Mexico State University, Las Cruces, NM 8003-8001, USA

e-mail: shahab@nmsu.edu

E. A. Butcher

e-mail: eab@nmsu.edu duction in patients with leukemia, chaotic time-delay systems have been employed in numerous other practical applications in engineering, biology, economy, and other disciplines [2-4]. The enhanced complexity of chaotic dynamics has played a significant role in the progress made in many of those applications.

It is well known that chaos in autonomous time continuous nonlinear systems can occur when the system has an order of greater than two and in non-autonomous time continuous nonlinear systems when it has an order of greater than one. However, in $1996 \mathrm{Lu}$ and He [5] showed that chaos can occur even in simple scalar first order delayed nonlinear (piecewise linear) dynamical systems with delay. Moreover, yet another significant difference of time delay systems compared with ODEs is that these systems can produce chaotic attractors with an arbitrarily large number of positive Lyapunov exponents (LEs). Therefore, scalar time-delay systems represent a major class of dynamical systems that exhibit hyperchaos.

Just to name a few out of the wide variety of applications chaotic time-delay systems can be pertinent to, a couple of examples are mentioned here. It has been demonstrated [6] that infinite dimensional chaotic dynamical systems can be used to forecast the fluctuation in the share market behavior. Another instance of chaotic time-delayed applications is in secure communication where an information signal containing some messages is transmitted using a chaotic signal as a broadband carrier. It is believed that the level of security can be even more improved if hyperchaotic modulation is used instead of a low-dimensional chaotic barrier [7]. For some examples of secure communication via hyperchaotic modulation the reader is referred to [8,9]. Another possible application is in chaotic interrogation for damage detection in structural systems, in which previous work $[10,11]$ has so far used only chaotic and hyperchaotic ODEs. 
There has been a few number of studies reported in the literature [12-15] concerning parameter identification in linear time delay systems. Parameter estimation of nonlinear timevarying DDEs with constant delay from fully and partially available data has been studied by Deshmukh [16] wherein an ideal case of no external random disturbance was considered. In a previous work of the current authors [17], estimation of parameters and states of stochastic non-chaotic delay differential equations having time-varying coefficients and constant delay from noise-corrupted incomplete measurements is studied. Also, in another study by the current authors [18], the method used in [17] is extended to include the estimation of the delay bounds and parameters in delayed vibratory systems with distributed delay modeled using delayed integro-differential equations (DIDEs). Parameter estimation in chaotic time delay systems has also been discussed in a number of studies before. Parameter and time delay estimation of a scalar time-delay chaotic system from noise-corrupted measurements through synchronization is explored by Rakshit et al. [19] wherein a least square approach is used to derive a system of differential equations which governs the temporal evolution of the parameters. A combination of synchronization based on dynamical feedback with an adaptive evolution for the unknown parameters is used by $\mathrm{Lu}$ and Cao [20] to estimate the unknown parameters for a second order delayed chaotic neural network with time-varying delay. Tang and Guan [21] studied the problem of estimating time delay and parameters of time-delayed firstorder scalar chaotic systems by first converting the problem into an optimization problem with a suitable objective function and applying a particle swarm optimization algorithm. Tang and Guan in another study [22] estimated the time delay and parameters of identical first-order scalar chaotic delayed systems by converting the problem to a multi-dimensional optimization problem and using a differential evolution algorithm. Sun and Yang [23] exploited chaos synchronization for parameter identification of chaotic delayed systems with varying time-delay through using an adaptive feedback controller based on the Razumikhin condition and the invariance principle of functional differential equations in the framework of Lyapunov-Krasovskii theory. However, among the aforementioned studies on parameter estimation of chaotic time delay systems only in $[20,23]$ a time-varying delay is taken into account. Nevertheless, in none of those studies a noise-corrupted measurement is considered. Additionally, the effect of uncertainty in the process (model) has always been neglected in studying parameter estimation of chaotic time delay systems.

In the current study a novel approach for simultaneous estimation of states, delay and parameters of chaotic and hyperchaotic delayed systems with constant or timevarying delay is proposed through exploiting continuous time approximation (CTA) and optimal stochastic filtering. Also in this paper an innovative technique is proposed to approximately compute the Lyapunov exponents of a delayed nonlinear system with the goal of determining the parameter combinations that results in chaotic or hyperchaotic systems. Specifically, this study explores stochastic estimation of parameters and states of chaotic DDEs having time-varying delay from a noise-corrupted measurement. In the case of chaotic DDEs with constant delay, simultaneous estimation of states, parameters and delay is also considered in this paper. To account for the effect of uncertainties of the model, an additive stochastic term is considered in the process. The proposed approach involves first discretizing the delay differential equation with a set of ordinary differential equations (ODEs) using the Chebyshev spectral CTA (CSCTA). Then, the problem of parameter estimation in the resulting ODE system is represented as an optimal filtering problem using a state augmentation technique. Finally, using an extended Kalman-Bucy filter the unknown parameters of the chaotic DDE are estimated from a noise-corrupted measurement of the states.

Major differences between the current study and the previous paper [17] of the current authors are as follows: in the previous study the delays were considered to be constant, while in this study parameter estimation in delayed systems with time-varying delays is also studied. However, the most important difference perhaps is that the DDEs considered in [17] were not chaotic whereas in the current study estimation is performed for both chaotic and hyperchaotic DDEs. The estimation problem is much more challenging in case of chaotic and hyperchaotic DDEs as opposed to linear or non-chaotic nonlinear DDEs studied in [17]. The major complexity that the chaotic nature of the DDE adds to the estimation problem is that CSCTA no longer provides accurate solutions. While the linear and nonlinear DDEs studied in [17] assumed that the model used in the process function was accurate and the only source of uncertainty was the process noise, in case of chaotic or hyperchaotic DDEs the finite-dimensional approximation based on CSCTA includes a source of uncertainty that increases dramatically over time. This is due to the characteristic known as 'sensitivity to infinitesimal perturbations'. While this results in the CSCTA approximate solution diverging after a short time, the resulting dynamics is an accurate projection of the infinite-dimensional chaotic attractor onto the corresponding finite-dimensional space assumed by CSCTA. This characteristic allows for CSCTA and the extended Kalman-Bucy filter to still deliver accurate estimates of constant parameters and delay, as is seen in the examples. Additionally, this paper focuses on the problem of delay estimation in chaotic DDEs which is not considered in the previous work of the current authors [17]. The problem of estimating the delay from measurements in a time-delayed system is known to be significantly more challenging than is the estimation of other para- 
meters. The major difference between the current paper and the other previous paper [18] of the current authors is that in [18] simultaneous estimation of states, parameters and delay upper bound of the distributed delay in linear and nonlinear time delayed systems with distributed delay described as DIDE's is investigated from noise corrupted measurements. While in this current study, as well as in [17], estimation in time delayed systems with discrete delay described as DDE's is considered. A different approach for CTA needs to be used when the delayed system is in DIDE form (distributed delay) than the one applicable to the DDE form of delayed systems (discrete delay).

The paper is organized as follows: In Sect. 2 it is shown how the CTA can be used for approximating nonlinear DDEs with discrete time-varying delays by an equivalent set of ODEs. A novel technique to approximately compute the LEs of DDEs using CSCTA is introduced in Sect. 3. Section 4 describes how the parameter estimation problem of DDEs can be handled in form of an optimal filtering problem taking advantage of CSCTA. In Sect. 5, the extended Kalman-Bucy filter is presented as an approach to deal with the resulting optimal filtering problem. Finally, in Sect. 6, the approach is implemented for state, parameter and delay estimation on various forms of the time-delayed Lorenz and delayed Hopfield neural network system including chaotic and hyperchaotic cases with constant and time-varying delays.

\section{Spectral CTA}

A DDE with a discrete time-varying delay can be described in the general form as

$\dot{\mathbf{x}}(t)=\boldsymbol{g}(\mathbf{x}(t), \mathbf{x}(t-\tau(t)), t, \mathbf{a})$,

$\mathbf{x}(t)=\varphi(\theta), \quad t_{m} \leq t=\theta \leq 0$

where $\mathbf{x} \in \mathbb{R}^{n}$ is a $n$-dimensional state vector, $\tau(t)$ $\left(0 \leq \tau(t) \leq \tau_{m}\right)$ is the time-varying delay, $\boldsymbol{\varphi}(t)$ is the history vector function defined on the interval $t \in\left[t_{m}, 0\right]$, and $t_{m}=\min _{t \geq 0}(t-\tau(t))$. This form of nonlinear delayed system is a very general one and includes all well-known delayed systems such as Ikeda system [24], Mackey and Glass system [25], Hopfield delayed neural network [26], delayed Duffing system [27], Delayed Lorenz system [28], BAM neural network [24], cellular neural network [29], etc. We assume the nonlinear DDE of Eq. (1) has $r$ unknown parameters represented by vector a to be estimated. In the context of functional analysis, the above DDE can be represented as an abstract ODE which in turn is a representation of the evolution of an initial function in a Banach space, i.e.

$$
\begin{aligned}
\dot{\mathbf{Y}}(t) & =\widetilde{\mathbb{A}}(t) \mathbf{Y}(t)+\mathbb{G}(\mathbf{Y}(t), t, \mathbf{a}) \\
\widetilde{\mathbb{A}}(0) \varphi & =\frac{d \boldsymbol{\varphi}}{d \theta}, \quad t_{m} \leq \theta<0
\end{aligned}
$$

$$
\begin{aligned}
\widetilde{\mathbb{A}}(0) \varphi+\mathbb{G}(\boldsymbol{\varphi}, 0, \mathbf{a}) & =\boldsymbol{g}(\mathbf{x}(0), \mathbf{x}(-\tau(0)), t, \mathbf{a}), \\
\theta & =0
\end{aligned}
$$

where $\mathbf{Y}(t)$ is an infinite-dimensional vector, $\widetilde{\mathbb{A}}(t)$ is a linear time-varying operator and $\mathbb{G}(\mathbf{Y}(t), t, \mathbf{a})$ is a time-varying infinite-dimensional nonlinear vector function of $\mathbf{Y}(t)$. The main idea behind CTA is that the infinite-dimensional vectors $\mathbf{Y}(t)$ and $\mathbb{G}(\mathbf{Y}(t), t, \mathbf{a})$ and the operator $\widetilde{\mathbb{A}}(t)$ can be approximated by finite-dimensional ones. Further information on the relation between the domain and the spectrum of the solution operator can be found in [30-34]. As shown and discussed in [35], spectral differentiation has a major advantage over finite difference differentiation $[32,34]$ in its "spectrally accurate" exponential convergence characteristics.

A finite-dimensional approximation to $\mathbf{Y}(\mathrm{t})$ is now defined as

$$
\begin{aligned}
\mathbf{Y}(t) & \cong\left[\mathbf{Y}_{1}^{\mathrm{T}}(t), \mathbf{Y}_{2}^{\mathrm{T}}(t), \ldots, \mathbf{Y}_{N+1}^{\mathrm{T}}(t)\right]^{\mathrm{T}} \\
\mathbf{Y}_{i}(t) & =\mathbf{x}\left(t-\tau_{i-1}\right), \quad i=1, \ldots, N+1
\end{aligned}
$$

where $\tau_{i-1}=\frac{\tau}{2}\left(1-t_{i-1}\right)$ and $t_{i-1}=\cos \left(\frac{(i-1) \pi}{N}\right)$ are the unevenly spaced points corresponding to the extremum points of the Chebyshev polynomial of the first kind [36] of degree $N$ defined in the interval $[-1,1]$. Thus with the Chebyshev points being defined so, the number of collocation points will be $m=N+1$.

We now define a $m \times m$ Chebyshev spectral differentiation matrix $\mathbf{D}$ associated with the Chebyshev points. Assuming the rows and columns of $\mathbf{D}$ matrix are indexed from 0 to $N$, the entries of the matrix will be

$$
\begin{aligned}
D_{00} & =\frac{2 N^{2}+1}{6}, D_{N N}=-\frac{2 N^{2}+1}{6}, \\
D_{j j} & =\frac{-t_{j}}{2\left(1-t_{j}^{2}\right)}, j=1, \ldots, N-1 \\
D_{i j} & =\frac{c_{i}(-1)^{i+j}}{c_{j}\left(t_{i}-t_{j}\right)}, \quad i \neq j, \quad i, j=0, \ldots, N
\end{aligned}
$$

where $c_{i}=2$ for $i=0, N$, otherwise $c_{i}=1$. The $m n \times m n$ differential operator $\mathbb{D}$ (corresponding to $n$ first order DDEs) is defined as $\mathbb{D}=\mathbf{D} \otimes \mathbf{I}_{n}$ in which $\mathbf{I}_{n}$ is a $n \times n$ identity matrix and $\otimes$ denotes Kronecker product. Equation (2) can be approximated by initially replacing the first $n$ rows of $\mathbb{D}$ by zeros to approximate $\widetilde{\mathbb{A}}(t)$ and by inserting the vector field associated with Eq. (1) in the top $n$ rows of $\mathbb{G}()$, with the remaining rows equal to zero as

$$
\left[\begin{array}{c}
\dot{\mathbf{Y}}_{1}(t) \\
\dot{\mathbf{Y}}_{2}(t) \\
\vdots \\
\dot{\mathbf{Y}}_{m}(t)
\end{array}\right]=\left[\begin{array}{c}
\mathbf{0} \mathbf{0} \ldots \mathbf{0 0} \\
\frac{2}{\tau(t)}\left[\mathbb{D}^{(n+1: n m,:)}\right]
\end{array}\right]\left[\begin{array}{c}
\mathbf{Y}_{1}(t) \\
\mathbf{Y}_{2}(t) \\
\vdots \\
\mathbf{Y}_{m}(t)
\end{array}\right]
$$




$$
+\left[\begin{array}{c}
\boldsymbol{g}\left(\mathbf{Y}_{1}(t), \mathbf{Y}_{m}(t), t, \boldsymbol{a}\right) \\
\mathbf{0}_{n \times 1} \\
\vdots \\
\mathbf{0}_{n \times 1}
\end{array}\right]
$$

Note that the superscript $(n+1: n m,:)$ on $\mathbb{D}$ refers to the fact that only rows of $\mathbb{D}$ lying between $n+1$ and $n m$ are written into the remaining $n(m-1) \times n m$ elements of the matrix $\widetilde{\mathbb{A}}(t)$. The $\frac{2}{\tau(t)}$ factor in front of $\mathbb{D}$ in Eq. (5) is a time-varying normalization factor which accounts for the rescaling of the standard collocation expansion interval $[-1,1]$ to the timevarying interval $[0, \tau(t)]$. Regardless of whether the original DDE has constant or time-varying coefficients, the operator $\widetilde{\mathbb{A}}(t)$ will be time varying for a time-varying delay due to the time-varying normalization used. Therefore, the nonlinear DDE of Eq. (1) can be converted into a large system of ODEs using CSCTA in which the dimension of Eq. (5) depends on the order of the Chebyshev grid used. A larger grid generally results in better accuracy of the ODE approximation.

An important point remains to be clarified here. Since the delay is time-varying, the length of the interval $[0, \tau(t)]$ on which the continuous functions are mapped (Banach space) also is changing, and if a discretization is used that enforces a constraint of constant distances between the (equally or non-equally) spaced grid points then some points will obviously need to be added or subtracted as the delay varies. This will change the size of the matrix operator and will add to the complexity of the problem. To avoid this in our work, however, the spacing between the grid points varies with the delay itself using the time-varying normalization factor in order to keep the number of (unequally spaced) grid points constant.

\section{Computing Lyapunov exponents of a DDE}

One of the most important tools widely accepted to quantify chaos is that of Lyapunov exponents. There exist some algorithms to calculate the Lyapunov spectrum of continuous and differentiable ODEs in the literature. The algorithm developed separately by Benettin [43] and Shimada [44] is one of those algorithms which is later modified and improved in [37]. This algorithm, however, is not applicable to infinite-dimensional DDEs. An adaptation of the algorithm for ODEs approach is suggested for DDEs by Farmer [38] which is based on discretizing the infinite-dimensional DDE into a $N$-dimensional discrete mapping. Yet another alternative technique makes use of the projection of DDEs to ODEs. The Galerkin projection technique [39], for instance, is one of those techniques. The accuracy of this technique depends on the number of shape functions retained.
The approach applied in this study to obtain the Lyapunov exponents of a nonlinear delayed system from its governing DDE, is based on CTA discussed in Sect. 2. As discussed before, the governing DDE can be represented as an abstract ODE of the form of Eq. (2) in terms of an infinitedimensional state vector $\mathbf{Y}(t)$, and the operator $\widetilde{\mathbb{A}}(t)$. CTA can then be utilized to approximate the infinite-dimensional vector $\mathbf{Y}(t)$ and the operator $\widetilde{\mathbb{A}}(t)$ by finite-dimensional ones through discretization of the delayed part of the state vector and spectral differentiation. As mentioned before, spectral differentiation is implemented by fitting an approximating polynomial through the values of the function evaluated at a set of unevenly distributed grid points based on Chebyshev points, then differentiating the polynomial, and finally valuating the result at each grid point as a linear combination of the nodal function values. The rest of the approach after obtaining the finite-dimensional state vector is an adaption of the technique used in finite dimensions by Wolf [37]. Lyapunov exponents can be defined by the long-term evolution of the axes of an infinitesimal sphere of states. A fiducial trajectory (the center of the sphere) is defined by the action of the nonlinear equation of motion $\dot{\boldsymbol{X}}=f(\boldsymbol{X})$ on some posttransient initial conditions. On the other hand, the principal axes are defined by the evolution via the linearized equations $\delta \dot{\boldsymbol{X}}=[\mathrm{J}] \delta \boldsymbol{X}$ of an initially orthonormal vector frame $v_{0}^{1}, \ldots v_{0}^{n}$ anchored to the fiducial trajectory. To implement the procedure, the fiducial trajectory and the frame of orthonormal vectors are simultaneously integrated. Due to an additional singularity in a chaotic system, each vector tends to fall along the local direction of most rapid growth. The collapse of the vectors toward a common direction is avoided by repeated use of the Gram-Schimdt Reorthonormalization (GSR). The orientation-preserving property of GSR $(v \rightarrow v$ ) allows the integration of the vector frame as is required for spectral convergence. Each Lyapunov exponent $\lambda_{i}$ may then be computed directly from the mean rate of growth of the projection of vector $v_{i}$ on vector $\hat{v}_{i}$.

The procedure presented in this study for Lyapunov exponent calculation of DDEs is verified using the results of

Table 1 Convergence study for the Mackey-Glass DDE of Eq. (6)

\begin{tabular}{lll}
\hline$\tau$ & $m$ & $\lambda_{i=1,2, \ldots, 4}$ \\
\hline 17 & 20 & $0.0054,-0.0030,-0.0428,-0.0595$ \\
& 40 & $0.0049,-0.0034,-0.0432,-0.0609$ \\
& 60 & $0.0020,-0.0071,-0.0430,-0.0617$ \\
25 & 70 & $0.0027,-0.0070,-0.0430,-0.0620$ \\
& 20 & $0.0081,-0.0021,-0.0097,-0.0335$ \\
& 40 & $0.0076,-0.0028,-0.0133,-0.0375$ \\
& 60 & $0.0108,-0.0025,-0.0124,-0.0354$ \\
& 70 & $0.0111,-0.0026,-0.0121,-0.0357$ \\
\hline
\end{tabular}



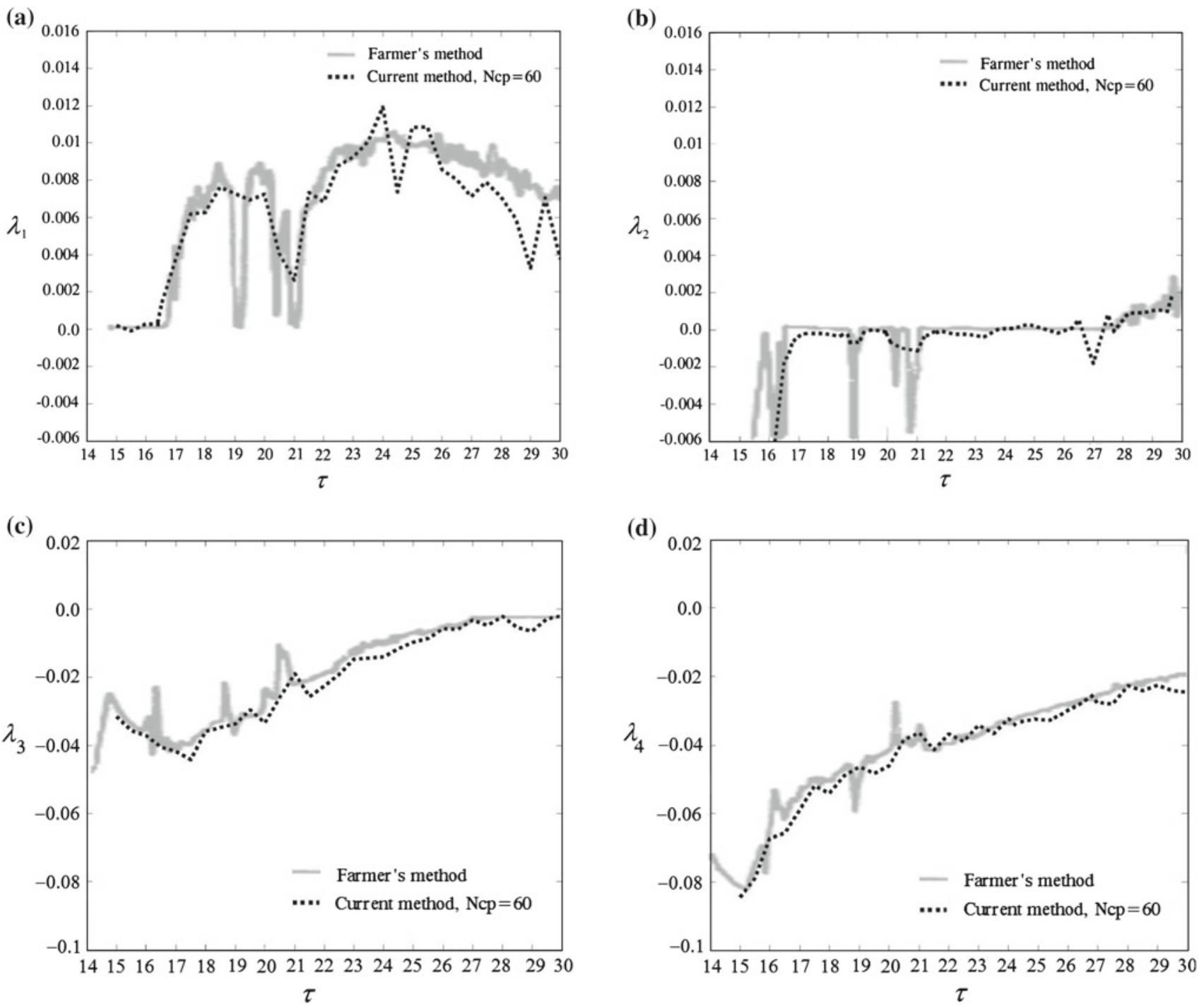

Fig. 1 The four largest Lyapunov exponents of the Mackey and Glass DDE of Eq. (6) versus the constant delay

the technique presented by Farmer [38]. The 4 largest Lyapunov exponents of the time-delayed system of Mackey and Glass for a delay varying from 15 to 35 are calculated using the current approach and the results are compared with those provided in Farmer's paper. A convergence study for some arbitrary values of $\tau$ within the range 15-30 tabulated in Table 1 shows that an acceptable convergence can be achieved by using 60 Chebyshev collocation points. The time-delayed system of Mackey and Glass is a scalar first order DDE of the following form which is first used to model blood production [1]:

$\dot{x}(t)=\frac{a x(t-\tau)}{1+x(t-\tau)^{c}}+b x(t)$

where $a=0.2, b=-0.1$ and $c=10$. The current state $x(t)$ represents the concentration of blood at time $t$ and the delayed state $x(t-\tau)$ is the concentration when the "request" for blood is made. In patients with leukemia, the delay $\tau$ may become excessively large and cause the concentration of blood to oscillate.

The results of comparing the four largest Lyapunov exponents of Eq.(6) obtained from the current method with that of Farmer's method are depicted in Fig. 1. As is clear from the figure, the results are in good agreement with those of Farmers method. Obviously, the accuracy of the proposed technique depends on the number of collocation points used. Also note that both the current method and Farmer's method use approximation techniques. The main advantage of the current technique over conventional discretization-based techniques such as Farmer's technique where equally-spaced discretization scheme is used can be attributed to the pseudospectral differentiation and consequently its exponential convergence characteristics. 


\section{Estimation problem in the form of optimal filtering}

A stochastic estimation problem in a delayed system obtains the best estimate of the current (and consequently the delayed) state as well as the unknown parameters of a stochastically-excited delayed system model from measured data that contains a random observation error. In addition to that, the stochastic estimation in this study is also applied to estimate the amount of the delay the stochastically-excited delayed system is experiencing from its noise-corrupted measured data. The estimation problem of a delayed system considered in this paper, can be formulated as an optimal continuous-time filtering problem in the form of a set of Ito stochastic delay differential equations as

$$
\begin{aligned}
& d \mathbf{x}(t)=\boldsymbol{f}(\mathbf{x}(t), \mathbf{x}(t-\tau(t)), \mathbf{a}(t), \mathrm{t}) d t+\mathbf{G}(t) d \boldsymbol{\beta}(t) \\
& d \mathbf{z}(t)=\boldsymbol{h}(\mathbf{x}(t), \mathbf{x}(t-\tau(t)), \mathrm{t}) d t+\mathbf{J}(t) d \boldsymbol{\eta}(t)
\end{aligned}
$$

where $\mathbf{x}(t) \in \mathbb{R}^{n}$ is the current Ito process, $\mathbf{x}(t-\tau(t)) \in \mathbb{R}^{n}$ is the delayed Ito process, $\mathbf{z}(t) \in \mathbb{R}^{\mathrm{q}}$ is the measurement process, $\mathbf{a}(t) \in \mathbb{R}^{r}$ is a vector of unknown parameters, $\boldsymbol{f}$ is the drift coefficient, $\mathbf{G}$ is the diffusion coefficient, $\boldsymbol{h}$ is the measurement model function, $\mathbf{J}$ is an arbitrary time-varying functions independent of $\mathbf{x}$, and $\boldsymbol{\beta}(t)$ and $\boldsymbol{\eta}(t)$ are independent Brownian motion additive stochastic processes with $E[d \boldsymbol{\beta}(t)]=\mathrm{E}[d \boldsymbol{\eta}(t)]=0, E\left[d \boldsymbol{\beta}(t) d \boldsymbol{\beta}^{T}(t)\right]=\mathbf{Q} d t$ and $E\left[d \boldsymbol{\eta}(t) d \boldsymbol{\eta}^{T}(t)\right]=\mathbf{R} d t$ where $E[]$ represents the expectation operator. Under certain conditions [40], the filtering problem can also be formulated in terms of the stationary zero-mean Gaussian white noise processes formally defined as $\mathbf{v}(t)=d \boldsymbol{\beta}(\mathrm{t}) / d t, \mathbf{w}(t)=d \boldsymbol{\eta}(\mathrm{t}) / d t$ and differential measurement $\mathbf{y}(t)=d \mathbf{z}(t) / d t$. Therefore, the filtering problem can be written in physical form by considering the drift function $f$ to be the nonlinear term $\mathbf{g}$ of Eq. (1).

$$
\begin{aligned}
& \dot{\mathbf{x}}(t)=\boldsymbol{g}(\mathbf{x}(t), \mathbf{x}(t-\tau(t)), \mathbf{a}(t), t)+\mathbf{G}(t) \mathbf{v}(t) \\
& \boldsymbol{y}(t)=\boldsymbol{h}(\mathbf{x}(t), \mathbf{x}(t-\tau), t)+\mathbf{J}(t) \mathbf{w}(t)
\end{aligned}
$$

where $\mathbf{v}(t)$ and $\mathbf{w}(t)$ are assumed to be both mutually independent and independent from the state and observation with constant covariance matrices of $\boldsymbol{Q}$ and $\boldsymbol{R}$, respectively, i.e. $\mathbf{v} \sim N(0, \boldsymbol{Q})$ and $\mathbf{w} \sim N(0, \boldsymbol{R})$. The stochastic term $\mathbf{v}(t)$ (the "process noise") can be considered to function as an approximation for the influence of the unknown dynamics of the process model. Eq. (8) defines a continuous-time statespace optimal filtering model. Under certain conditions, the optimal continuous-time filtering problem has a finite dimensional solution which leads to the Kalman-Bucy filter. The filtering problem can be represented in the context of parameter and delay estimation. Suppose that $\mathbf{a}(t)$ is unknown but is assumed to be constant. The Kalman-Bucy filter can be used to simultaneously estimate the states $\mathbf{x}(t)$, the parameters $\mathbf{a}(t)$, and the delay $\tau(t)$. The standard method for parameter estimation via filtering employs the so-called state augmen- tation method, in which the parameter vector a $(t)$ is included with the state vector while being constrained to have a zero rate of change, i.e.

$$
\begin{aligned}
\left\{\begin{array}{l}
\dot{\mathbf{x}}(t) \\
\dot{\mathbf{a}}(t)
\end{array}\right\} & =\left\{\begin{array}{l}
\boldsymbol{g}\left(\mathbf{x}, \mathbf{x}_{\tau}, \mathbf{a}, t\right) \\
\mathbf{0}
\end{array}\right\}+\left\{\begin{array}{l}
\mathbf{G}(t) \\
\mathbf{0}
\end{array}\right\} \mathbf{v}(t) \\
\mathbf{y}(t) & =\boldsymbol{h}\left(\mathbf{x}, \mathbf{x}_{\tau}, t\right)+\mathbf{J}(t) \mathbf{w}(t)
\end{aligned}
$$

where $\mathbf{x}_{\tau}$ denotes $\mathbf{x}(t-\tau(t))$. The parameter vector $\mathbf{a}(t)$ is assumed to initially have a Gaussian distribution with mean $\mathbf{a}_{0}$ and covariance $\boldsymbol{P}_{0}$. Note that there is no noise term in the equation for the unknown parameter dynamics. The reason is that the parameters are already assumed to be stationary. In order for the approach to be applicable, the DDE of the system needs to be expressed as an ODE. Using spectral CTA to convert the nonlinear DDE of Eq. (8) into a set of ODEs, the augmented state-space expression of the optimal filtering problem of Eq. (9) can be written as

$$
\begin{aligned}
& \left\{\begin{array}{c}
\dot{\mathbf{Y}}(t) \\
\dot{\mathbf{a}}(t)
\end{array}\right\}= \\
& \left\{\left[\begin{array}{ccccc}
\mathbf{0}_{n \times n} & \mathbf{0}_{n \times n} & \ldots & \mathbf{0}_{n \times n} & \mathbf{0}_{n \times n} \\
& \frac{2}{\tau(t)}[ & {\left[\mathbb{D}^{(n+1: 1: n m,:)}\right]} &
\end{array}\right] \mathbf{Y}(t)+\left[\begin{array}{c}
\boldsymbol{g}\left(\mathbf{Y}_{1}(t), \mathbf{Y}_{m}(t), t\right) \\
\mathbf{0}_{n(m-1) \times 1}
\end{array}\right]\right\} \\
& +\left\{\left[\begin{array}{c}
\mathbf{G}(t) \\
\mathbf{0}_{n(m-1) \times n} \\
\mathbf{0}_{r \times n}
\end{array}\right]\right\} \mathbf{v}(t) \\
& \mathbf{y}(t)=\boldsymbol{h}(\mathbf{Y}(t), t)+\mathbf{J}(t) \mathbf{w}(t)
\end{aligned}
$$

where $\mathbf{v}(t)$ and $\mathbf{w}(t)$ are zero-mean white Gaussian noise vectors respectively of dimension $n \times 1$ and $q \times 1$.

For delay estimation via filtering, two different approaches can be considered. In the first approach after using CSCTA to approximate the original DDE with a finite-dimensional ODE, the delay which appears as a normalization factor in the operator matrix $\widetilde{\mathbb{A}}(t)$ is treated as another parameter of the system. Assuming the delay to be constant (or possibly having a predetermined rate of change if not constant) and using state augmentation technique, the delay can be estimated via optimal filtering in a similar way as parameters are estimated. In the second approach, however, we employ a non-dimensional time $\tilde{t}=t / \hat{\tau}(t)$ through using transformation $t \rightarrow \tilde{t}$ and also writing the derivative $\frac{d}{d t}$ in terms of the new variable $\tilde{t}$. Note that $\hat{\tau}(t)$ is the estimate of the delay at instant $t$. Then after using CSCTA, the delay term in the normalization factor in the operator matrix $\widetilde{\mathbb{A}}(t)$ will vanish. Again using state augmentation technique, the time evolution of the unknown delay can be estimated via optimal filtering techniques. For the case when the delay is constant, the delay term in the new format of Eq. (10) will appear as coefficients of $\mathbf{g}$ as shown below. 


$$
\begin{aligned}
& \left\{\begin{array}{c}
\dot{\mathbf{Y}}(\tilde{t}) \\
\dot{\tau}(\tilde{t})
\end{array}\right\}=
\end{aligned}
$$

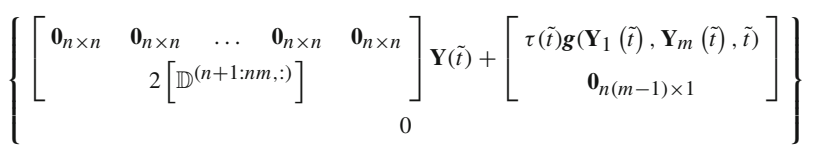

$$
\begin{aligned}
& +\left\{\left[\begin{array}{c}
\mathbf{G} \tilde{t} \\
\mathbf{0}
\end{array}\right]\right\} \mathbf{v}(\tilde{t}) \\
& \mathbf{y}(\tilde{t})=\boldsymbol{h}(\mathbf{Y}(\tilde{t}), \tilde{t})+\mathbf{J}(\tilde{t}) \mathbf{w}(\tilde{t})
\end{aligned}
$$

\section{Extended Kalman-Bucy filters}

Assuming the augmented delayed state to be incorporated in a finite-dimensional state $\boldsymbol{X}$, i.e. $\boldsymbol{X}^{\boldsymbol{T}}(t)=\left[\boldsymbol{Y}^{\mathrm{T}}(t), \mathbf{a}^{\mathrm{T}}(t)\right], \mathrm{Eq}$. (10) can be written as a nonlinear optimal filtering problem without delay in the Ito form

$$
\begin{aligned}
d \boldsymbol{X}(t) & =\mathcal{F}(\boldsymbol{X}(t), t) d t+\mathcal{G}(t) d \boldsymbol{\beta}(t) \\
d \mathbf{z}(t) & =\mathcal{H}(\boldsymbol{X}(t), t) d t+\mathbf{J}(t) d \boldsymbol{\eta}(t)
\end{aligned}
$$

where $E[d \boldsymbol{\beta}(t)]=\mathrm{E}[d \boldsymbol{\eta}(t)]=0, E\left[d \boldsymbol{\beta}(t) d \boldsymbol{\beta}^{T}(t)\right]=\boldsymbol{Q} d t$ and $E\left[d \boldsymbol{\eta}(t) d \boldsymbol{\eta}^{T}(t)\right]=\boldsymbol{R} d t$. In order for the Kalman-Bucy filter to be applicable to the nonlinear system, the dynamics needs to be linearized. Rather than linearizing about a reference trajectory, the extended Kalman-Bucy filter employs a linearization about the state estimate $\widehat{\boldsymbol{X}}$ which yields

$$
\begin{aligned}
\mathcal{F}(\boldsymbol{X}, t) & =\mathcal{F}(\widehat{\boldsymbol{X}}, t)+\widetilde{\boldsymbol{F}}(t)(\boldsymbol{X}-\widehat{\boldsymbol{X}})+\boldsymbol{r}_{\mathcal{F}}(\boldsymbol{X}, \widehat{\boldsymbol{X}}, t) \\
\mathcal{H}(\boldsymbol{X}, t) & =\mathcal{H}(\widehat{\boldsymbol{X}}, t)+\widetilde{\boldsymbol{H}}(t)(\boldsymbol{X}-\widehat{\boldsymbol{X}})+\boldsymbol{r}_{\mathcal{H}}(\boldsymbol{X}, \widehat{\boldsymbol{X}}, t)
\end{aligned}
$$

where $\boldsymbol{r}_{\mathcal{F}}()$ and $\boldsymbol{r}_{\mathcal{H}}()$ are higher order terms of the Taylor series and $\widetilde{\boldsymbol{F}}(t)$ and $\widetilde{\boldsymbol{H}}(t)$ are the Jacobian matrices obtained from linearization about the current estimates.

$$
\widetilde{\boldsymbol{F}}(t):=\left.\frac{\partial \mathcal{F}(\mathbf{X}, t)}{\partial \mathbf{x}}\right|_{\mathbf{x}=\widehat{\mathbf{x}}}, \quad \widetilde{\boldsymbol{H}}(t):=\left.\frac{\partial \mathcal{H}(\mathbf{X}, t)}{\partial \mathbf{x}}\right|_{\mathbf{x}=\hat{\mathbf{x}}}
$$

The propagation of the estimate based on extended Kalman-Bucy filter can be derived as

$$
d \widehat{\mathbf{X}}(t)=\mathcal{F}(\mathbf{X}, t) d t+\boldsymbol{K}(t)[d \mathbf{z}(t)-\mathcal{H}(\widehat{\mathbf{X}}, t) d t]
$$

where the gain matrix $\boldsymbol{K}(t)$ is

$$
\boldsymbol{K}(t)=\boldsymbol{P}(t) \widetilde{\boldsymbol{H}}^{T}(t) \boldsymbol{R}^{-1}
$$

The following Riccati differential equation propagates the error covariance $\boldsymbol{P}(t)$.

$$
\begin{aligned}
d \boldsymbol{P}(t)= & \widetilde{\boldsymbol{F}}(t) \boldsymbol{P}(t) d t+\boldsymbol{P}(t) \widetilde{\boldsymbol{F}}(t) d t \\
& +\mathcal{G}(t) \mathbf{Q}(\mathrm{t}) \mathcal{G}(t)^{T} d t-\boldsymbol{K}(t) \widetilde{\boldsymbol{H}}(t) \boldsymbol{P}(t) d t
\end{aligned}
$$

Considering the estimator given by Eqs. (15) to (17), it can be shown that for a uniformly detectible system with a bounded measurement and process noise, the estimation error $\boldsymbol{e}(t)=$ $\boldsymbol{X}(t)-\widehat{\boldsymbol{X}}(t)$ remains bounded if the initial estimation error is selected within a certain limit. This is summarized by the following theorem. Note that the time-dependence of some parameters is ignored in the theorem for ease of notation. The interested readers are referred to [41] for a detailed derivation of the conventional and extended Kalman-Bucy filter as well as the proof to the following theorem.

Theorem Consider a uniformly detectible nonlinear stochastic continuous system defined by Eq. (12) and also consider the extended Kalman-Bucy filter as described by Eqs. (15) to (22). Further suppose the following assumptions hold:

I. There exist real numbers $\delta \mathcal{F}>0, \delta \mathcal{H}>0$ and $\varepsilon>$ 0 such that for any $|\boldsymbol{X}(t)-\widehat{\boldsymbol{X}}(t)| \leq \varepsilon$ the nonlinear functions $\boldsymbol{r}_{\mathcal{F}}$ and $\boldsymbol{r}_{\mathcal{H}}$ in Eq. (13) are bounded:

$$
\begin{aligned}
\left\|\boldsymbol{r}_{\mathcal{F}}(\boldsymbol{X}, \widehat{\boldsymbol{X}}, t)\right\| & \leq \delta_{\mathcal{F}}\|\boldsymbol{X}(t)-\widehat{\boldsymbol{X}}(t)\|^{2} \\
\left\|\boldsymbol{r}_{\mathcal{H}}(\boldsymbol{X}, \widehat{\boldsymbol{X}}, t)\right\| & \leq \delta_{\mathcal{H}}\|\boldsymbol{X}(t)-\widehat{\boldsymbol{X}}(t)\|^{2}
\end{aligned}
$$

II. The covariance matrices of the noise terms are bounded for every $t \geq 0$ :

$$
\begin{aligned}
\mathcal{G} \boldsymbol{Q} \mathcal{G}^{T} & \leq \kappa_{q} \boldsymbol{I} \\
\mathbf{J} \boldsymbol{R} \mathbf{J}^{T} & \leq \kappa_{r} \boldsymbol{I}
\end{aligned}
$$

Then the estimation error $\boldsymbol{e}(t)$ remains bounded if the initial estimate error satisfies

$$
\begin{aligned}
& \|e(0)\|^{2} \leq \frac{\kappa_{\text {noise }} \lambda_{\max }\left(P^{-1}\right)}{\lambda_{\max }(\Omega)-\delta}, \quad \delta<\lambda_{\max }(\Omega) \\
& \text { where } \boldsymbol{\Omega}=-\boldsymbol{H}^{T} \boldsymbol{K}^{T} \boldsymbol{P}^{-1}-\boldsymbol{P}^{-1} \boldsymbol{Q} \boldsymbol{P}^{-1}, \kappa_{\text {noise }}=\kappa_{q}- \\
& \lambda_{\text {max }}^{2}(\boldsymbol{K}) \kappa_{r}, \delta=2 \delta_{\mathcal{F}} \lambda_{\max }(\boldsymbol{\Pi})-2 \delta_{\mathcal{H}} \lambda_{\max }(\boldsymbol{\Pi}) \lambda_{\max }(\boldsymbol{K}) \\
& \text { and } \boldsymbol{\Pi} \text { is defined by } \boldsymbol{\Pi}(t) \boldsymbol{P}(t)=I .
\end{aligned}
$$

\section{Estimation results}

\subsection{Time-delayed Lorenz oscillator}

Since 1963, when Edward Lorenz presented his familiar three-dimensional dynamical system as a truncation of the infinite-dimensional Navier-Stokes equations, the chaotic behavior observed in an infinite-dimensional system has always been explored through studying a low-dimensional attractor that is believed to capture the dominant dynamics in the infinite-dimensional system. Investigating chaos in an infinite-dimensional dynamical system, however, has only recently been closely investigated. In order to motivate subsequent discussion on infinite-dimensional chaotic systems, we study the dynamical behavior of the time-delayed Lorenz oscillator [28] which is governed by 

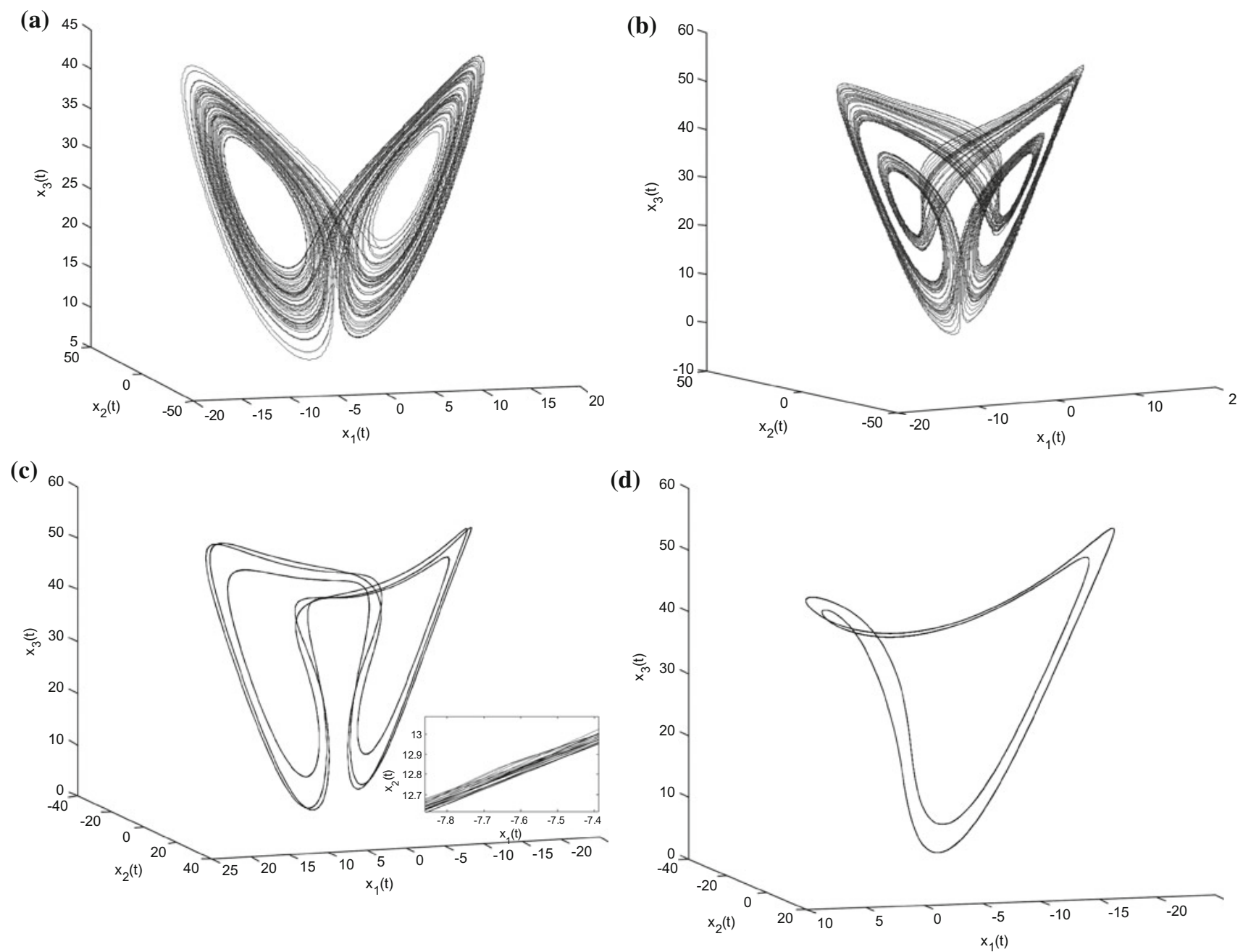

Fig. 2 Phase portrait of time-delayed Lorenz attractor of the system of Eq. (21) with $\sigma=10, \rho=28, \beta=8 / 3$ and a constant delay a $\tau=0$, $\mathbf{b} \tau=\frac{1}{6}, \mathbf{c} \tau=1, \mathbf{d} \tau=1.2$
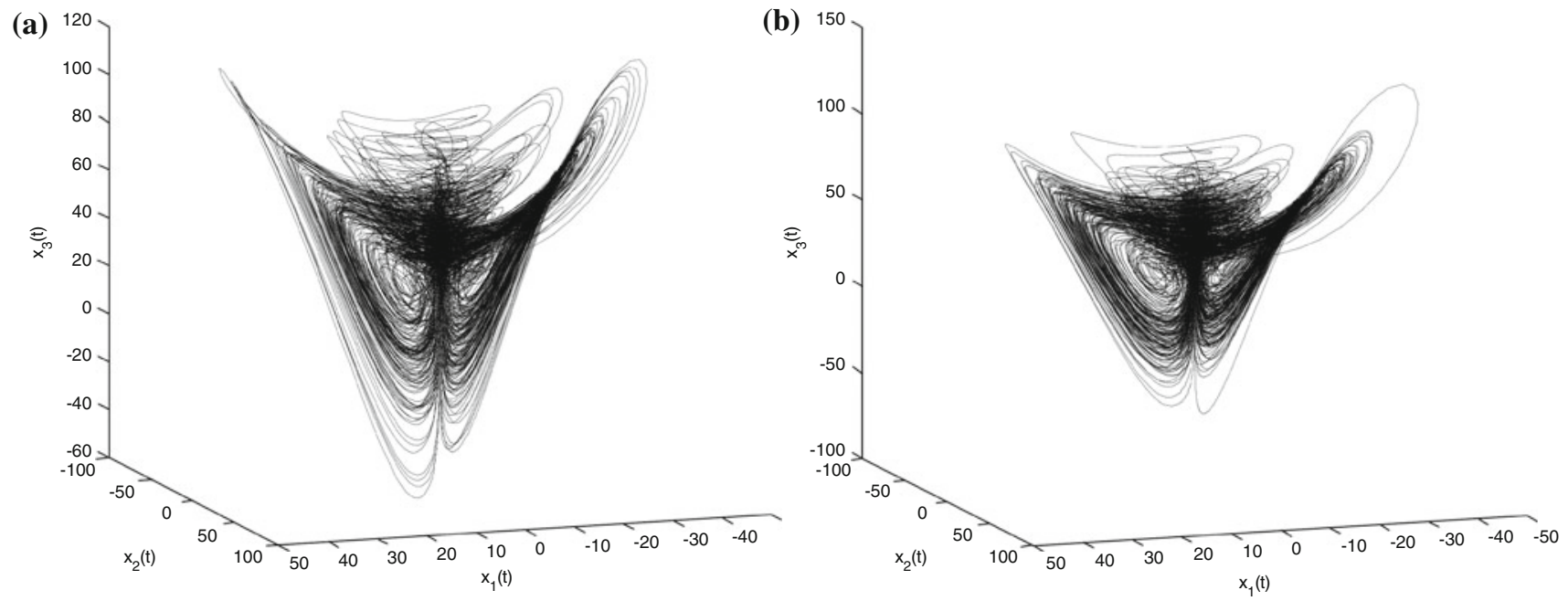

Fig. 3 Phase portrait of hyperchaotic attractors of delayed Lorenz system of Eq. (21) with $\sigma=10, \rho=28, \beta=8 / 3$ and a constant time-delay a $\tau=4, \mathbf{b} \tau=5.2$ 
$\dot{x}_{1}(t)=\sigma\left(x_{2}(t)-x_{1}(t)\right)$

$\dot{x}_{2}(t)=\rho x_{1}(t)-x_{2}(t)-x_{1}(t) x_{3}(t)$

$\dot{x}_{3}(t)=x_{1}(t) x_{2}(t)-\beta x_{3}(t-\tau(t))$

where $\mathbf{x}(t)$ and $\mathbf{x}(t-\tau(t))$ are the current and the delayed states at time $t$ and $\sigma, \rho$ and $\beta$ are constant parameters. Note that $\tau(t)$ (if non-zero) can be a positive constant or a nonnegative function of time. When $\tau=0$ the system becomes the familiar Lorenz chaotic oscillator for which it is known that a two-scroll chaotic attractor exists for $\sigma=10, \rho=28$ and $\beta=8 / 3$. As mentioned before, a common approach which has long been widely used to determine the complete Lyapunov spectrum of an attractor (non-delayed system) from its differential equations can be found in [42,43]. Based on this method, the Lyapunov spectrum of the non-delayed chaotic Lorenz system has one positive and one zero Lyapunov exponents as $\lambda_{i=1,2,3}^{C}=0.9056,0.0000,-14.5723$. The well-known phase portrait of the chaotic attractor when $\tau=0$ is shown in Fig. 2a.

When $\tau(t)>0$, the Lyapunov exponents are not as easily attainable as for the non-delayed case. The approach presented in Sect. 3 can be used to approximately find the Lyapunov spectrum from the DDE of the system. Phase portraits of the Lorenz DDE in Eq. (21) for some non-zero constant delays are depicted in Fig. 2b-d. Case b is chaotic (with a single positive Lyapunov exponent), while cases $\mathrm{c}$ and $\mathrm{d}$ represents brief transition to (quasi-) periodicity.

The delayed Lorenz system of Eq. (21) can also be hyperchaotic (with more than one positive Lyapunov exponent) without any further change in the equations and just by setting appropriate values of $\tau(t)$. The phase portraits of hyperchaotic attractors for two constant values of $\tau$ are depicted in Fig. 3.

As an example of the case when $\tau$ is a function of time, we consider a delay function which is used in [20,23], i.e.

$\tau(t)=\frac{\tau_{0} e^{\epsilon_{1} t}}{1+e \epsilon^{2 t}}$

The phase portraits of two chaotic attractors for the Lorenz DDE in Eq. (21) with the time-varying delay in Eq. (22) for two sets of values of $\tau_{0}, \epsilon_{1}$ and $\epsilon_{2}$ are depicted in Fig. 4. Note that in Fig. 4a the delay function is monotonically decreasing, so the phase portrait rather resembles the non-delayed Lorenz attractor. In Fig. 4b, however, a monotonically increasing delay function leads to a much more complicated attractor.

The technique proposed in Sect. 3 is utilized for calculating the Lyapunov exponents of the time-delayed Lorenz system of Eq. (21) while a constant delay is used. Again after performing a convergence study for multiple delay points, the number of collocation points is chosen to be 60 . The four largest LEs of the time-delayed Lorenz system of Eq. (21) are plotted versus delay for a range of constant values of $\tau \in[0.1,10]$ in Fig. 5 . It can be seen that the system exhibits a variety of different behavior including chaotic, hyperchaotic (with one and two positive Lyapunov exponents), and even a non-chaotic behavior depending on the value of the delay. The numerical values of the four largest Lyapunov exponents of the system for some values of constant delay are listed in Table 2 .

\subsubsection{Chaotic Lorenz DDE with constant delay}

Consider the Lorenz DDE of Eq. (21) with a constant delay of $\tau=\frac{1}{6}$ whose chaotic attractor is depicted in Fig. 2b. Assuming the parameters of the delayed oscillator $\mathbf{a}=[\sigma \rho \beta]$ to be unknown, the Lorenz DDE of Eq. (21) can be written in the form of filtering problem of Eq. (8) as

$$
\begin{aligned}
\dot{\mathbf{x}}(t) & =\boldsymbol{g}(\mathbf{x}(t), \mathbf{x}(t-\tau), \mathbf{a}(t), t)+\mathbf{G}(t) \mathbf{v}(t) \\
& =\left\{\begin{array}{l}
a_{1}\left(x_{2}(t)-x_{1}(t)\right) \\
a_{2} x_{1}(t)-x_{2}(t)-x_{1}(t) x_{3}(t) \\
x_{1}(t) x_{2}(t)-a_{3} x_{3}(t-\tau(t))
\end{array}\right\}+\mathbf{G}(t) \mathbf{v}(t) \\
\mathbf{y}(t) & =\boldsymbol{h}(\mathbf{x}(t), \mathbf{x}(t-\tau), t)+\mathbf{J}(t) \mathbf{w}(t)=\mathbf{x}(t)+\mathbf{J}(t) \mathbf{w}(t)
\end{aligned}
$$

where $\boldsymbol{G}(t)$ and $\boldsymbol{J}(t)$ are assumed to be identity matrices and the true values of the vector of unknown parameters are assumed to be $\mathbf{a}=\left[\begin{array}{ll}a_{1} & a_{2} \\ a_{3}\end{array}\right]=\left[\begin{array}{lll}10 & 28 & 8 / 3\end{array}\right]$. The current states are assumed to be directly measurable thus the measurement function $\boldsymbol{h}=\mathbf{x}(t)$ is used. The initial point of the oscillator is chosen to be at $\mathbf{x}=[0.94,0.27,17.27]$ which is a point on the attractor. In order to simulate the measurements, the Lorenz DDE of Eq. (23) with true parameters is integrated using the dde23 function in Matlab. Then, the resulting time history after being corrupted by an additive zero-mean Gaussian noise w $(t)$ having a covariance of $\boldsymbol{R}$ is applied in the simulation as the measured states. In order to account for uncertainties in the model a zero-mean Gaussian noise $\mathbf{v}(t)$ with a covariance of $\boldsymbol{Q}$ is added to the process state.

The sufficient number of collocation points for CSCTA based on a convergence study shown in Fig. 6 a is $m=20$. A comparison between the response of the Lorenz DDE with $\tau=\frac{1}{6}$ integrated by the Matlab dde23 integrator with that obtained from integrating the set of ODEs acquired from CSCTA with 20 collocation points using an 8th order Runge-Kutta integrator is shown in Fig. 6b. The comparison clearly shows that the CSCTA approximation diverges from the true solution within a short time. Increasing the number of collocation points does not help. Thus, while CSCTA is already shown to deliver good approximations of the solution in case of highly nonlinear delayed systems in [17], this discrepancy can obviously be attributed to the chaotic nature of the delayed oscillator and sensitive dependence on initial conditions. Therefore, a model based on CSCTA contains enormous uncertainty in case of chaotic 

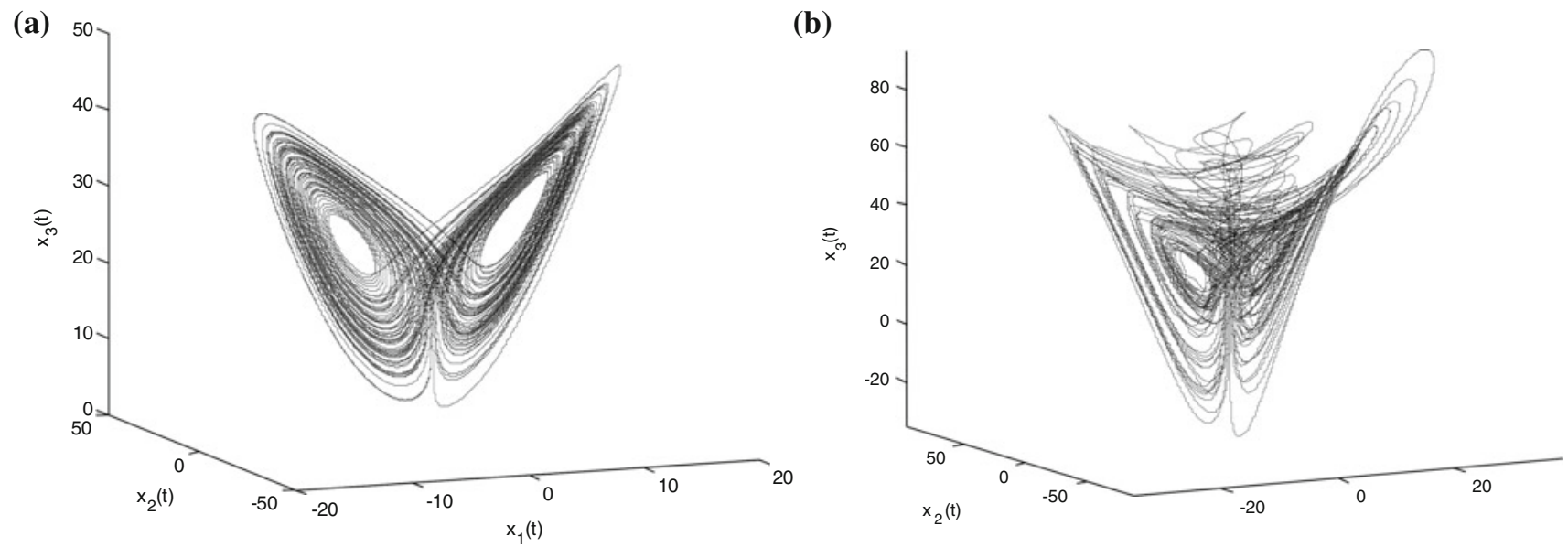

Fig. 4 Phase portrait of chaotic attractor of delayed Lorenz system of Eq. (21) with $\sigma=10, \rho=28, \beta=8 / 3$ and a time-varying delay of Eq. (22) $\mathbf{a} \tau_{0}=0.01, \epsilon_{1}=1$ and $\epsilon_{2}=2 ; \mathbf{b} \tau_{0}=0.01, \epsilon_{1}=1.1$ and $\epsilon_{2}=1$

(a)

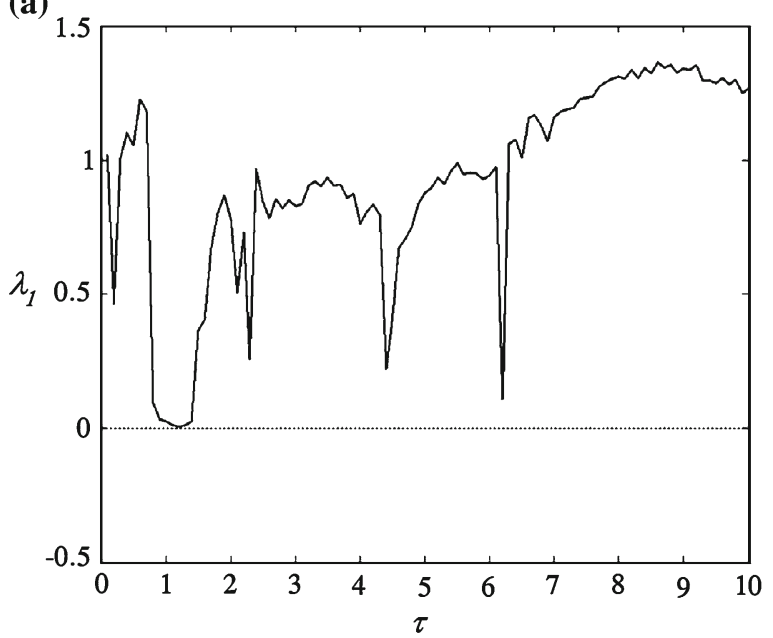

(c)

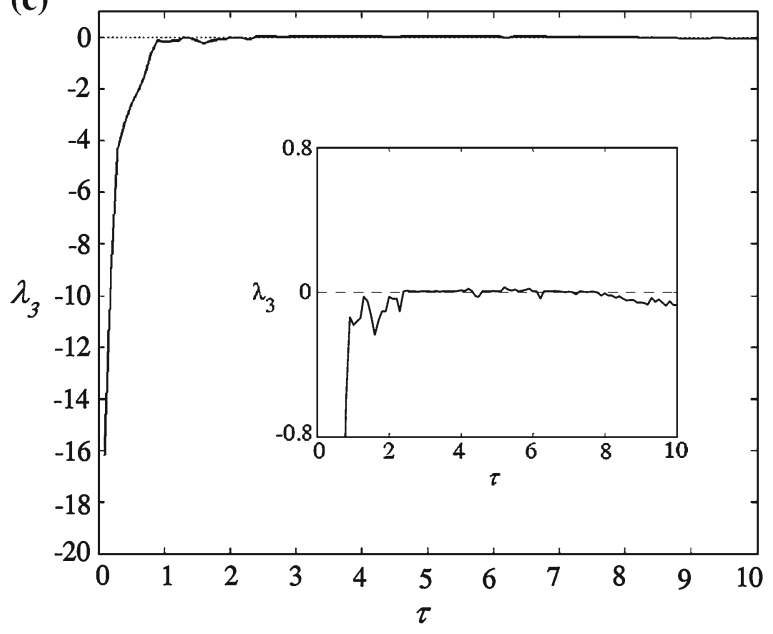

(b)

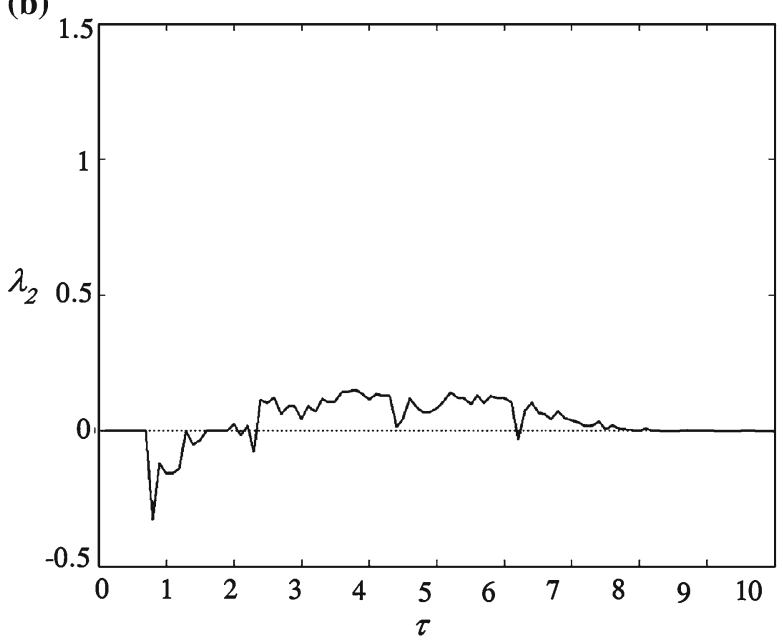

(d)

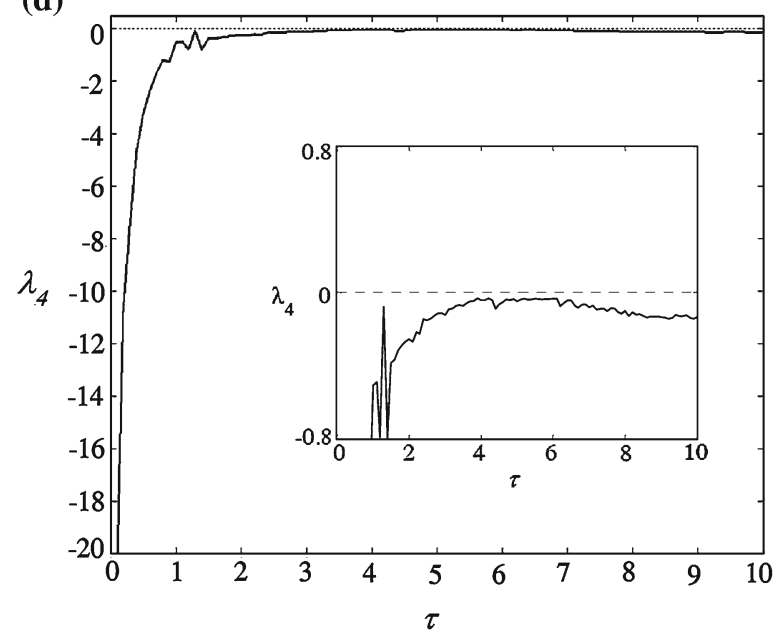

Fig. 5 The four largest Lyapunov exponents of time-delayed Lorenz system of Eq. (21) with $\sigma=10, \rho=28, \beta=8 / 3$ versus the delay 
Table 2 Lyapunov exponents of Lorenz DDE for different values of delay

\begin{tabular}{llll}
\hline$\tau$ & $m$ & $\lambda_{i=1,2, \ldots, 4}$ & Behavior \\
\hline 0.01 & 20 & $0.91,0.00,-14.64,-268.6$ & Chaotic \\
0.166 & 60 & $0.66,0.00,-10.08,-12.65$ & Chaotic \\
1.00 & 80 & $0.00,-0.16,-0.17,-0.48$ & Quasi-periodic limit cycle \\
1.2 & 60 & $0.00,-0.13,-0.80,-1.01$ & Quasi-periodic limit cycle \\
4.00 & 60 & $0.88,0.13,0.00,-0.02$ & Hyperchaotic (2PLEs) \\
5.2 & 80 & $0.91,0.15,0.02,0.00$ & Hyperchaotic (3PLEs) \\
\hline
\end{tabular}

Lorenz oscillator and model predictions are not reliable. This source of uncertainty in the model obviously has larger impact than the process noise. Despite the uncertainty of the model, the resulting dynamics from CSCTA is a projection of the infinite-dimensional attractor on the corresponding finite-dimensional subspace assumed by CSCTA, and hence the Extended Kalman-Bucy Filter (EKBF) may still be applied to simultaneously estimate all three states and all three parameters of the system from a noise-corrupted measurement. The estimation filtering sequence is initiated with a first guess for the augmented estimated state to be $50 \%$ deviated from the true values. The initial error covariance matrix, $\mathbf{P}_{0}$, the covariance of the measurement noise, $\boldsymbol{R}$, and the covariance of the process noise, $\boldsymbol{Q}$ are listed in Table 3 . The estimated states obtained by extended Kalman-Bucy filter along with the true state and the noise-corrupted measurements are shown in Fig. 7. Also the estimation errors for all three estimated states along with error covariance envelopes $(1 \sigma)$ are plotted versus time in Fig. 7. The root mean square errors (RMSE) of the estimated states, along with the estimated values of the unknown parameters after $5 \mathrm{~s}$ are listed in Table 3. As is clear from the results, the approach is capable of estimating the states and all parameters of the chaotic Lorenz DDE with a good accuracy from a highly uncertain model, a rough initial guess, and a highly noise-corrupted measurement.

\subsubsection{Hyperchaotic Lorenz DDE with constant delay}

Chaos intrinsically induces a high sensitivity to infinitesimal perturbations to the system. The extent of the sensitivity of a dynamical system to infinitesimal perturbation is characterized by the rate of separation of infinitesimally close trajectories (Lyapunov exponent) and also by the number of dimensions along which this expansion occurs (number of positive Lyapunov exponents). In a chaotic attractor the dynamics experience a one-dimensional expansion, that is, the dynamics expand as a line segment (one positive Lyapunov exponent). However, by definition hyperchaos is the dynamics associated with a chaotic attractor with more than one-dimensional expansion (more than one positive Lyapunov exponent). Thus, if a hyperchaotic attractor has three positive Lyapunov exponents, its dynamics expands as a volume element. This indicates that in a hyperchaotic oscillator the degree of the characteristic sensitivity under longterm integration is significantly higher than that of a chaotic oscillator. Therefore, the CSCTA-based model is expected to exhibit a higher degree of uncertainty when applied to approximate a hyperchaotic infinite-dimensional dynamics rather than that of an infinite-dimensional chaotic dynamics. It is noted that, unlike the case of a chaotic ODE such as the standard Lorenz oscillator in which an additional state is required to initiate hyperchaos, in the case of chaotic DDEs due to the infinite-dimensional characteristic of DDEs, only a slight change of parameter values is generally necessary.

In order to verify the estimation technique under the challenging conditions of hyperchaos, the Lorenz DDE with $\tau=4$ and $\tau=5.2$ which lead to hyperchaotic attractors with 2 and 3 positive Lyapunov exponents, respectively (whose attractors are depicted in Fig. 3) are considered here for state and parameter estimation. The procedure is repeated once with a delay of $\tau=4$ and again with a delay of $\tau=5.2$ using a measurement noise, a process noise and an initial error covariance as shown in Table 3. The estimation filtering sequences in both cases are initiated with a first guess for the augmented state to be $50 \%$ deviated from the true values. The estimated states obtained by the extended Kalman-Bucy filter along with the estimation errors for all three estimated states for the case of $\tau=4$ are shown in Fig. 8 and for the case of $\tau=5.2$ are shown in Fig. 9. The RMSE of the estimated states and the estimated values of the unknown parameters after $5 \mathrm{~s}$ for both $\tau=4$ and $\tau=5.2$ are listed in Table 3. Note that the average error of the estimated parameters for the hyperchaotic case with 3 positive Lyapunov exponents ( $\tau=5.2$ ), despite the lower level of noise applied, is larger than for the hyperchaotic case with 2 positive Lyapunov exponents $(\tau=4)$. Also, the average error of the estimated parameters for the hyperchaotic case with 2 positive Lyapunov exponents $(\tau=4)$, is higher larger than that of the chaotic case $(\tau=1 / 6)$ studied in previous section. This clearly indicates the higher degree of uncertainty of the model due to the larger number of positive Lyapunov exponents in the case hyperchaotic Lorenz DDEs. 

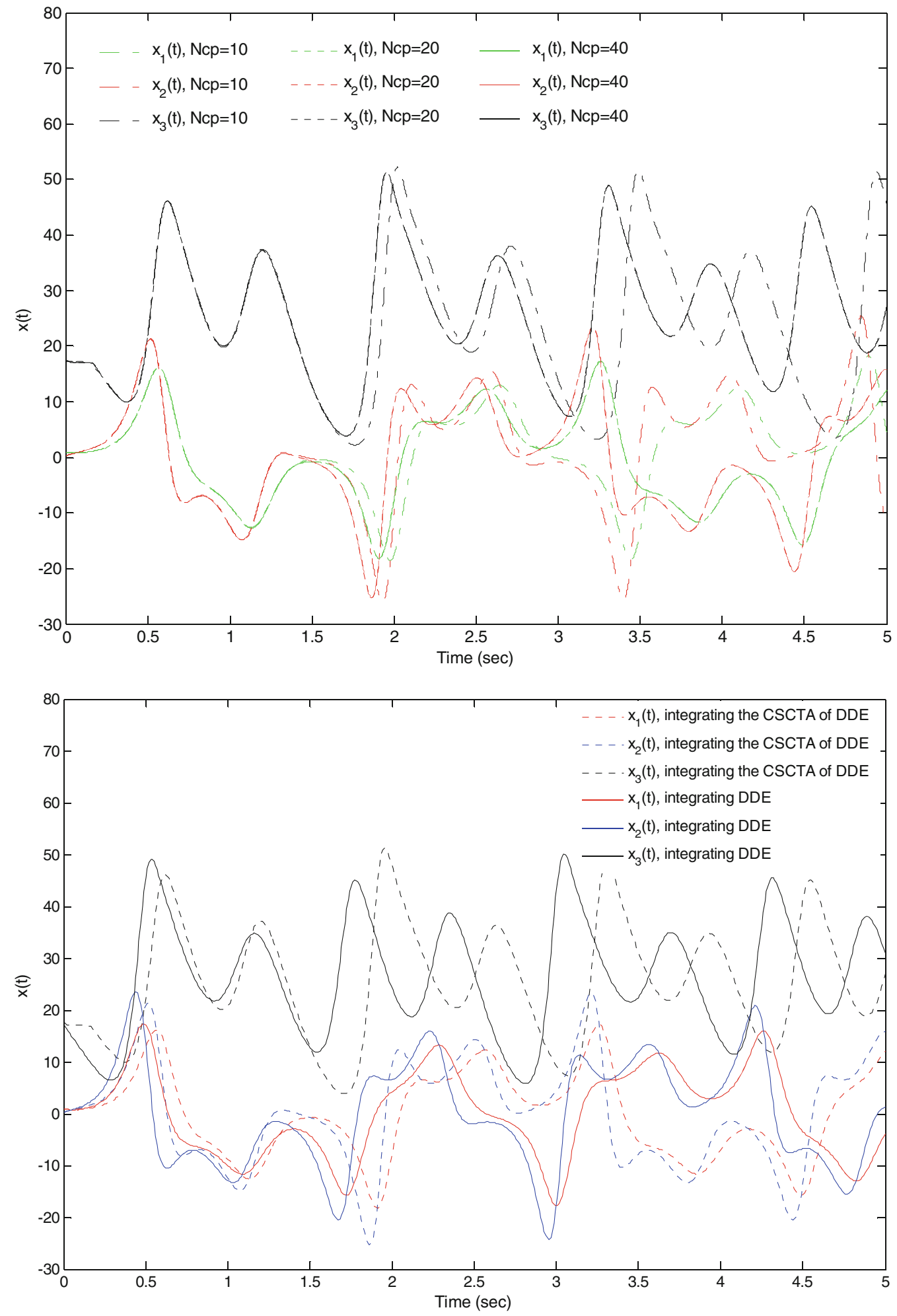

Fig. 6 Chaotic delayed Lorenz oscillator of Eq. (21) $(\sigma=10, \rho=$ $28, \beta=8 / 3, \tau=1 / 6)$ initiated at $\mathrm{x}=[0.94,0.27,17.27]$ : $\mathbf{a}$ convergence study to find the sufficient number of collocation points $m$ required for CSCTA, b comparison between the integrated response obtained from the CSCTA of the DDE with those obtained from the original DDE to show the uncertainty in the predictions of the CSCTAbased model 


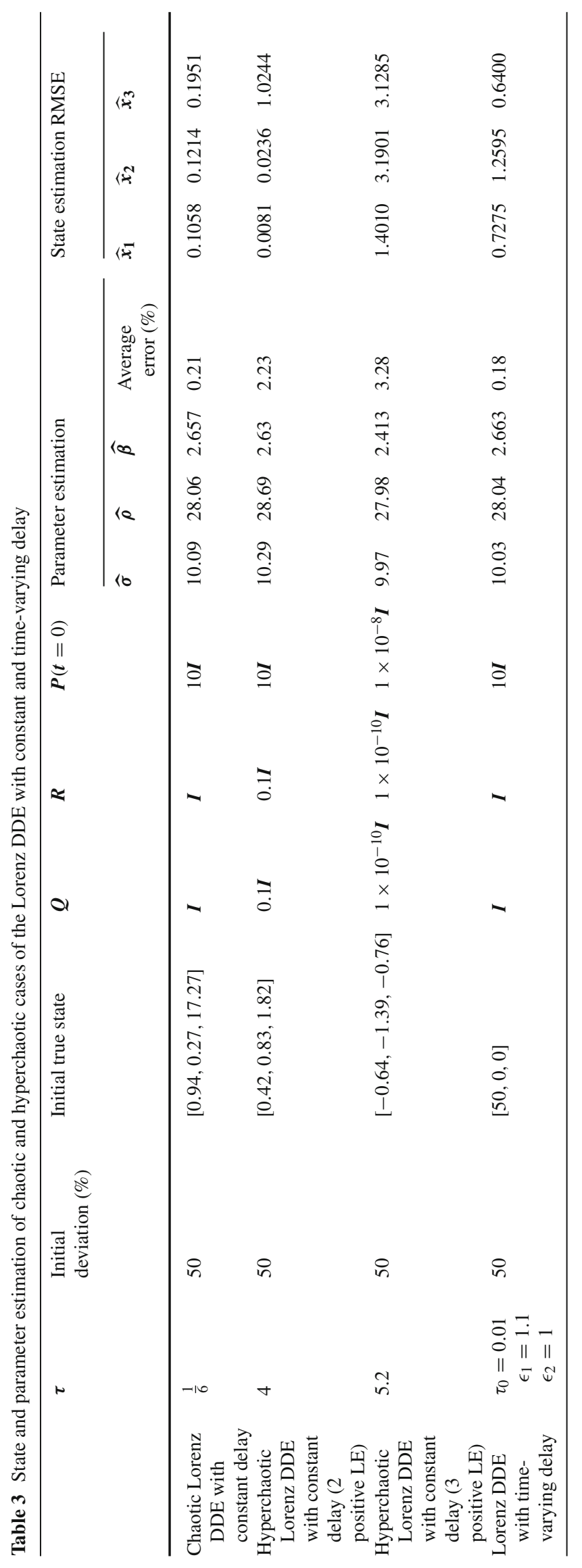



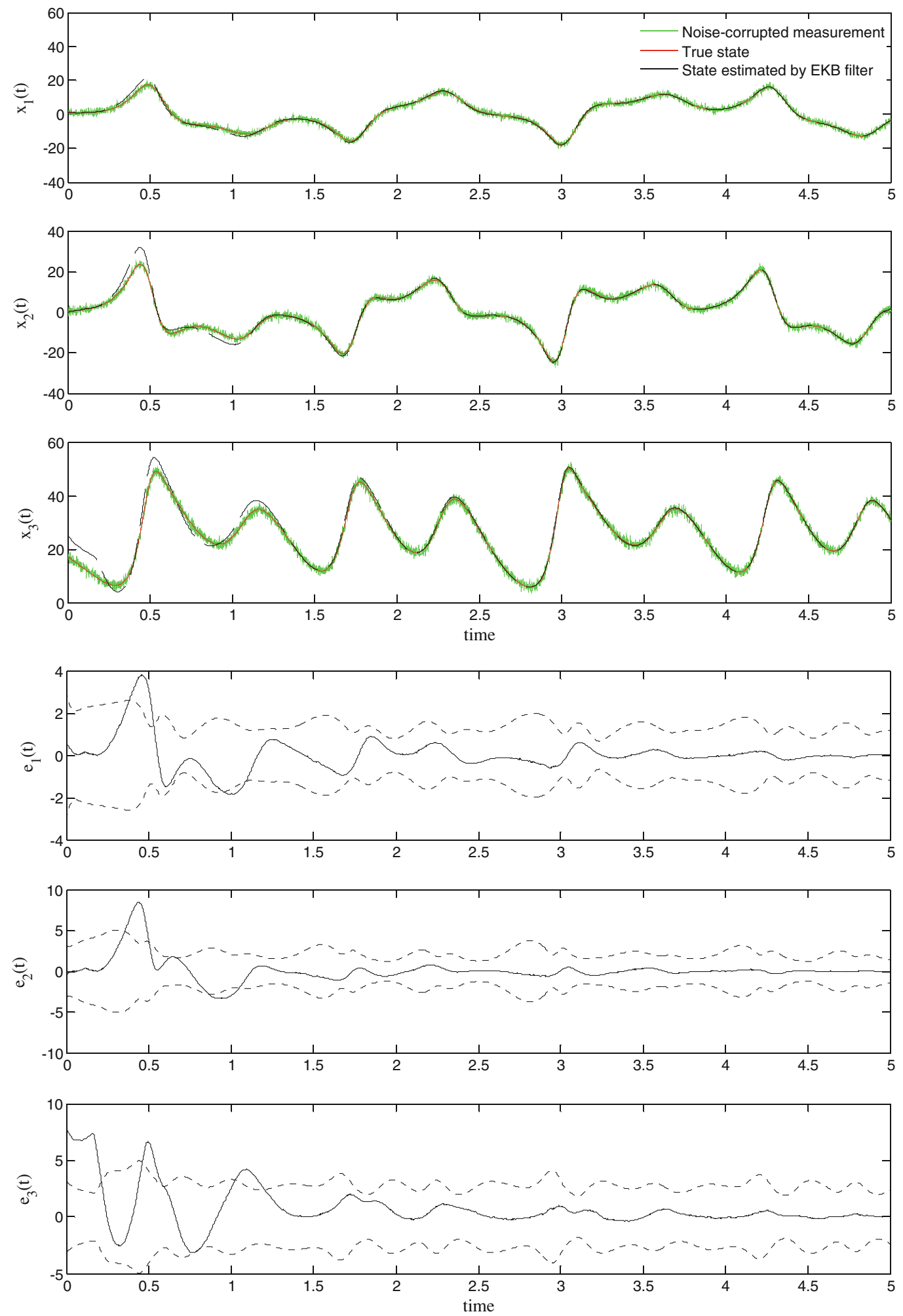

Fig. 7 States of the chaotic case of the Lorenz DDE ( $\sigma=10, \rho=28, \beta=8 / 3, \tau=1 / 6)$ initiated at $\mathrm{x}=[0.94,0.27,17.27]$ estimated using $\mathrm{EKBF}$ along with the estimation error (solid line) and $1 \sigma$ error covariance envelope (dotted line) of EKBF 

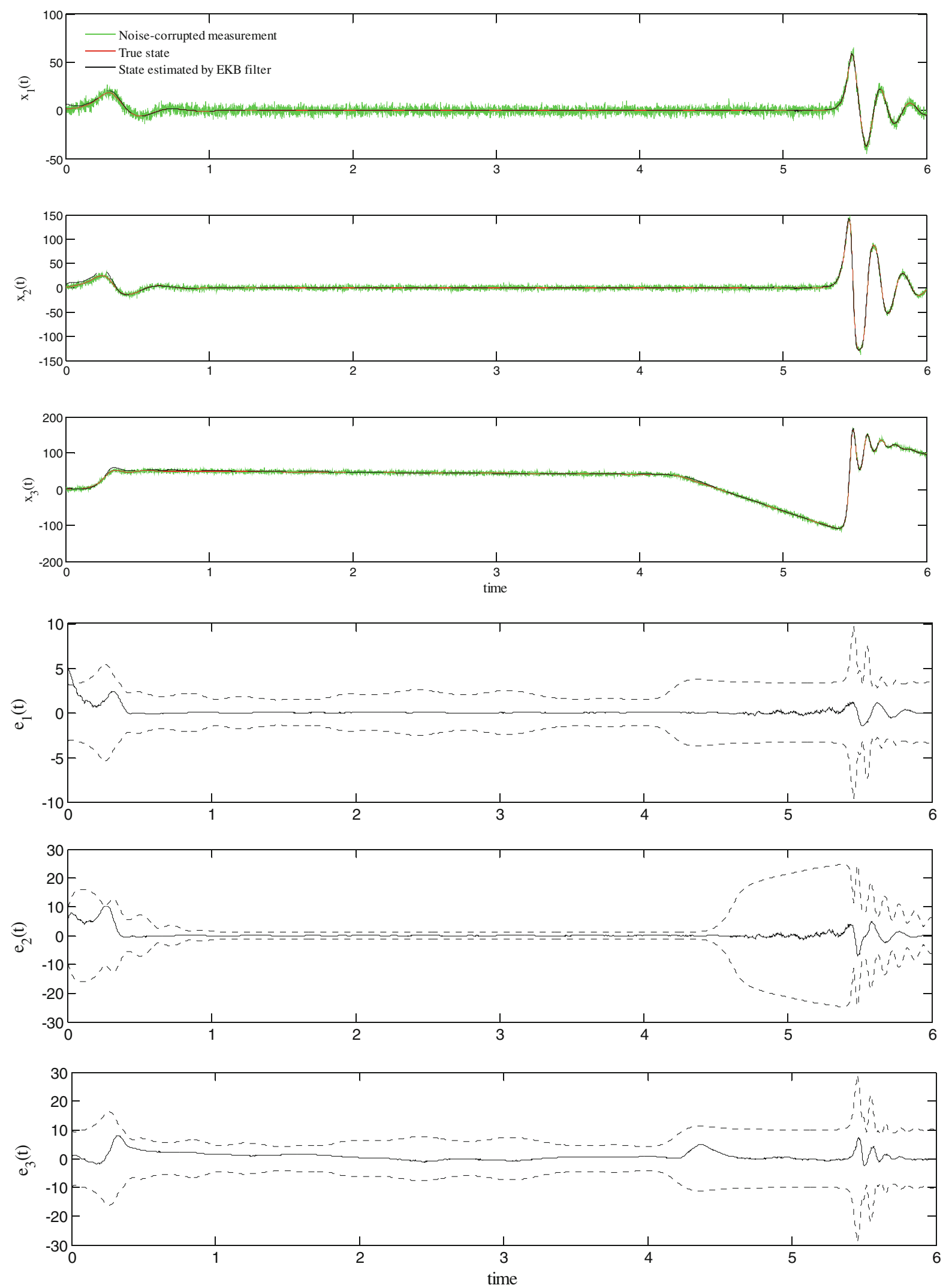

Fig. 8 States of the hyperchaotic case of delayed Lorenz system $(\sigma=10, \rho=28, \beta=8 / 3, \tau=4)$ initiated at $\mathrm{x}=[2,1,1]$ estimated using $\mathrm{EKBF}$ along with the estimation error (solid line) and $1 \sigma$ error covariance envelope (dotted line) of EKBF 

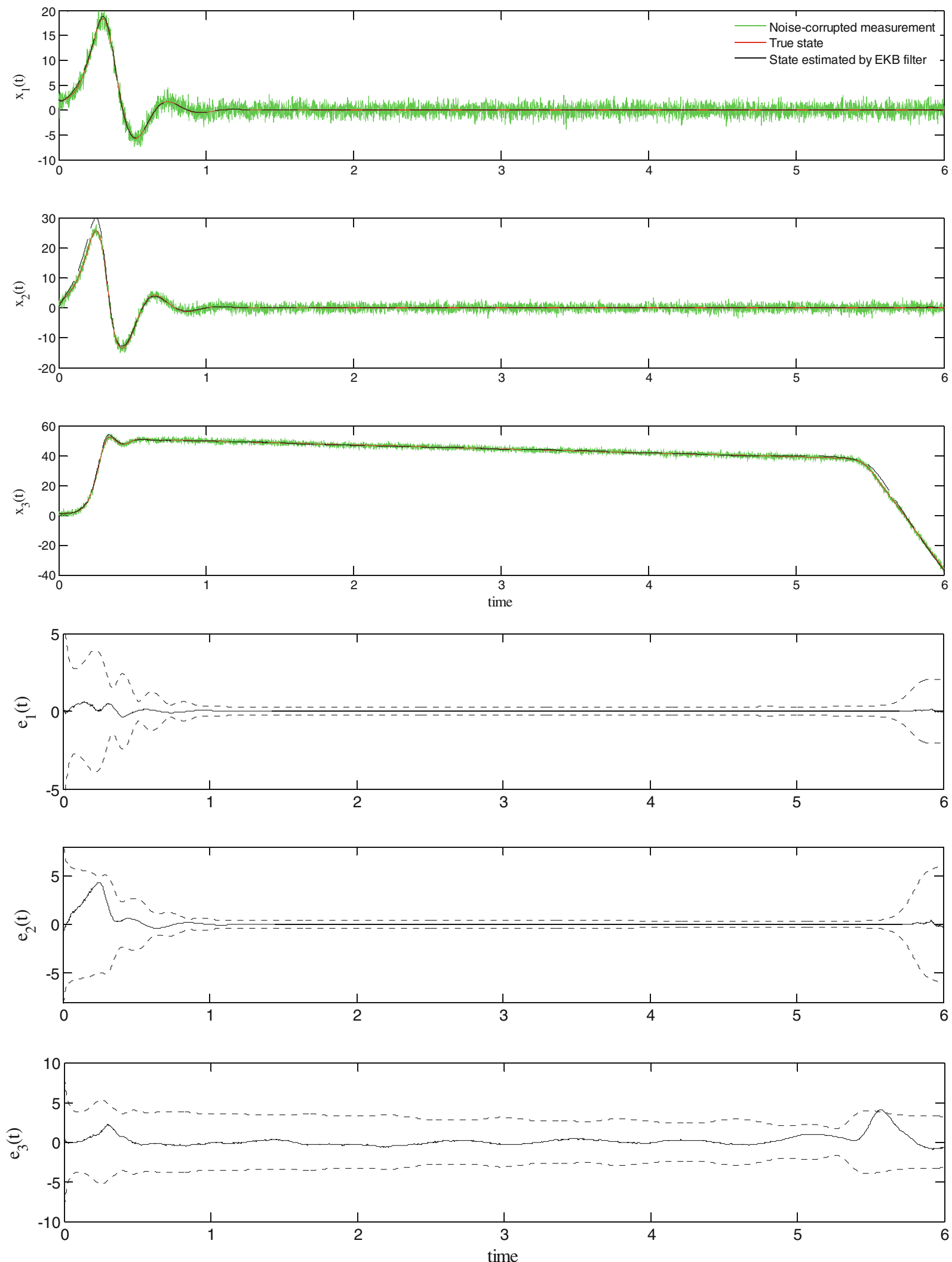

Fig. 9 States of the hyperchaotic case of the Lorenz DDE $(\sigma=10, \rho=28, \beta=8 / 3, \tau=5.2)$ initiated at $\mathrm{x}=[2,1,1]$ estimated using EKBF along with the estimation error (solid line) and $1 \sigma$ error covariance envelope (dotted line) of EKBF 

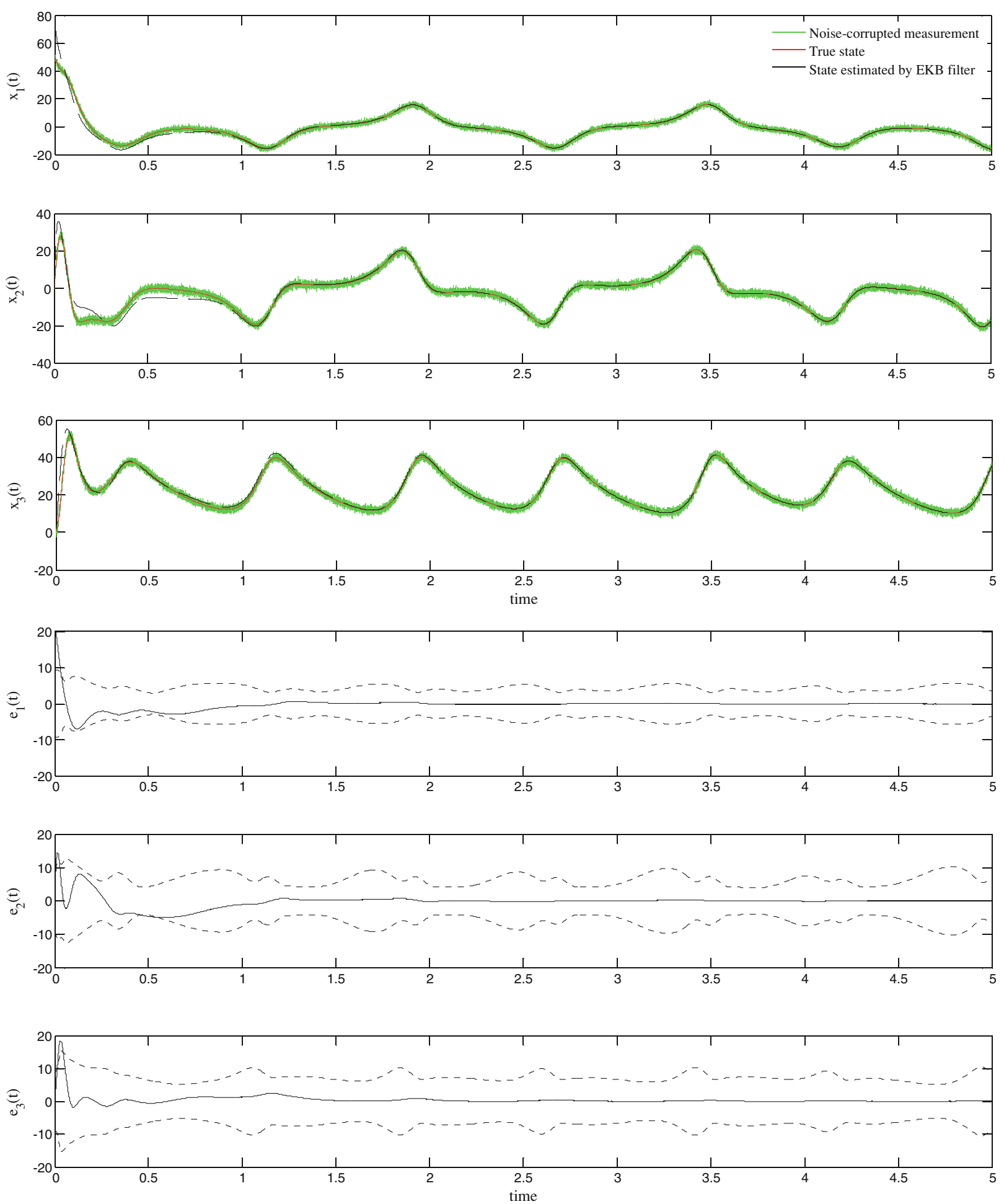

Fig. 10 States of the Lorenz DDE with the time varying delay of Eq. (22) $\left(\sigma=10, \rho=28, \beta=\frac{8}{3}, \tau_{0}=0.01, \epsilon_{1}=1.1, \epsilon_{2}=1\right)$ estimated using $\mathrm{EKBF}$ along with the estimation error (solid line) and $3 \sigma$ error covariance envelope (dotted line) of EKBF

\subsubsection{Lorenz DDE with nonlinear time-varying delay}

The capability of the proposed estimation technique in estimating the parameters and states of a time delayed system with time-varying delay is assessed using the Lorenz DDE of Eq. (21) with the time-varying delay of Eq. (22). The values of coefficients $\tau_{0}, \epsilon_{1}$ and $\epsilon_{2}$ used for this simulation are $\tau_{0}=0.01, \epsilon_{1}=1.1$ and $\epsilon_{2}=1$ which corresponds to the 
(a)

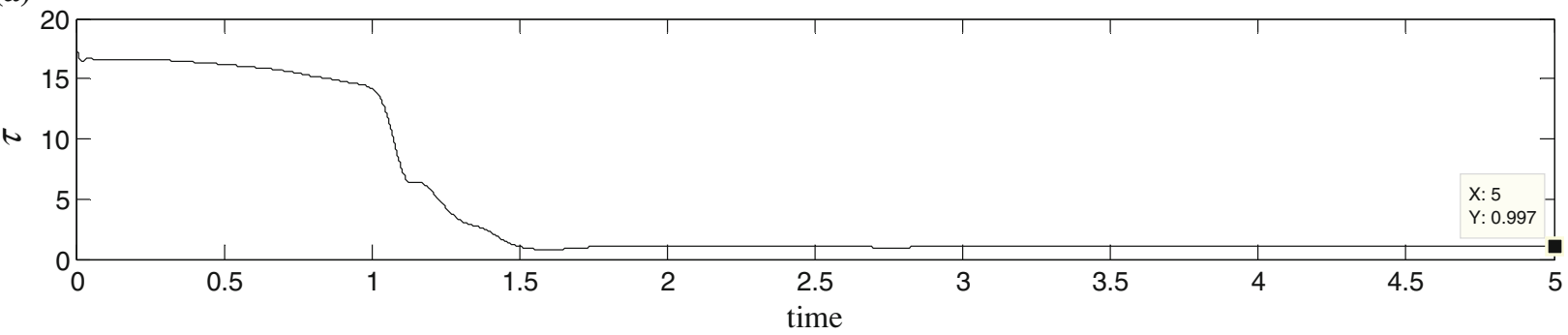

(b)

n

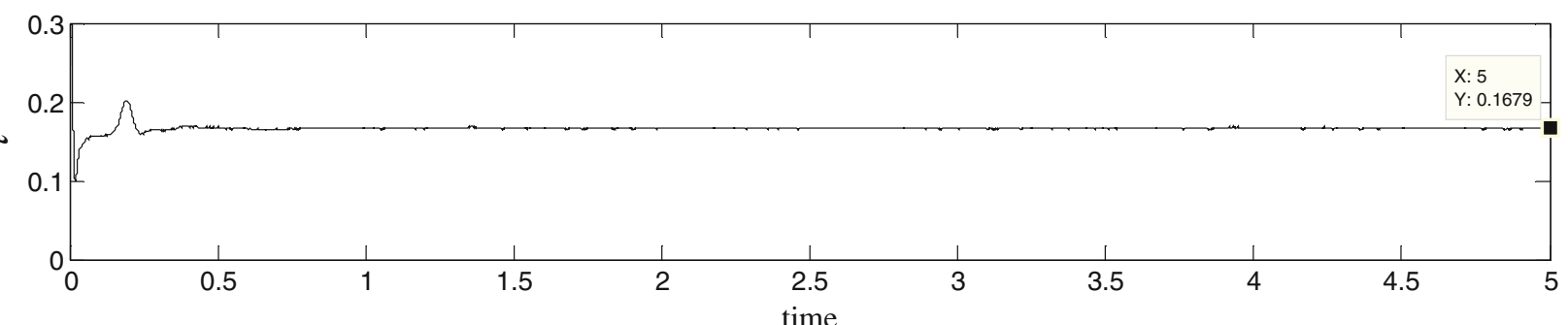

(c)

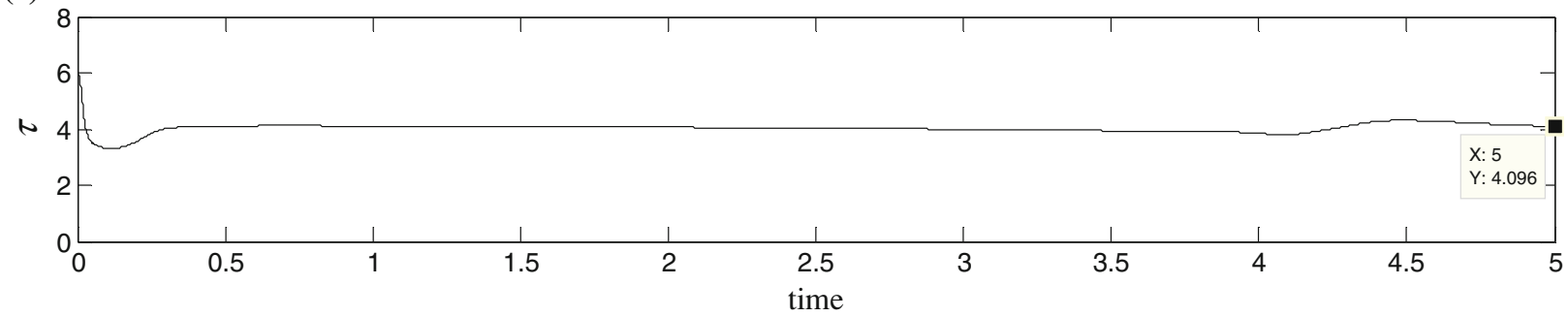

Fig. 11 Unknown constant delay of the quasi-periodic, chaotic and hyperchaotic cases of the Lorenz DDE with $\sigma=10, \rho=28, \beta=\frac{8}{3}$ estimated using EKBF. The initial guess used for the delay are $\mathbf{a} \hat{\tau}_{0}=10, \mathbf{b} \hat{\tau}_{0}=1 / 4, \mathbf{c} \hat{\tau}_{0}=6$. true values of the delay are $\mathbf{a}=1, \mathbf{b} \tau=1 / 6, \mathbf{c} \tau=4$

Table 4 State and delay estimation of quasi-periodic, chaotic and hyperchaotic cases of the Lorenz DDE with constant delay

\begin{tabular}{|c|c|c|c|c|c|c|c|c|c|c|}
\hline & \multirow[t]{2}{*}{$\tau$} & \multirow[t]{2}{*}{ Initial deviation (\%) } & \multirow[t]{2}{*}{$Q$} & \multirow[t]{2}{*}{$\boldsymbol{R}$} & \multirow[t]{2}{*}{$P(t=0)$} & \multicolumn{2}{|c|}{ Delay estimation } & \multicolumn{3}{|c|}{ State estimation RMSE } \\
\hline & & & & & & $\hat{\boldsymbol{\tau}}$ & Error $(\%)$ & $\widehat{x}_{1}$ & $\widehat{x}_{2}$ & $\widehat{x}_{3}$ \\
\hline $\begin{array}{l}\text { Chaotic case of } \\
\text { the Lorenz } \\
\text { DDE (with } \\
\text { constant delay) }\end{array}$ & $\frac{1}{6}$ & 50 & $0.01 I$ & $0.01 I$ & $0.1 I$ & 0.1679 & 0.78 & 0.0155 & 0.0226 & 0.0184 \\
\hline $\begin{array}{l}\text { Quasi-periodic } \\
\text { case of the } \\
\text { Lorenz DDE } \\
\text { (with constant } \\
\text { delay) }\end{array}$ & 1.00 & 1,000 & $I$ & $I$ & $10 I$ & 0.997 & 0.3 & 1.8785 & 2.9130 & 7.3430 \\
\hline $\begin{array}{l}\text { Hyperchaotic } \\
\text { case of the } \\
\text { Lorenz DDE } \\
\text { (with constant } \\
\text { delay) }\end{array}$ & 4.00 & 50 & $0.01 I$ & $0.01 I$ & $0.1 I$ & 4.096 & 2.4 & 0.1760 & 0.2043 & 1.6009 \\
\hline
\end{tabular}

attractor depicted in Fig. 4b. Again the vector of unknown

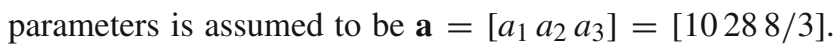
In order to simulate the measurements, the Lorenz DDE of Eq. (21) with the time-varying delay of Eq. (22) and with true parameters is integrated in Matlab this time using the ddesd function which is designed to handle DDE integration with time or state-dependant delay. The integrated response is used as the measured states in the simulation after adding 

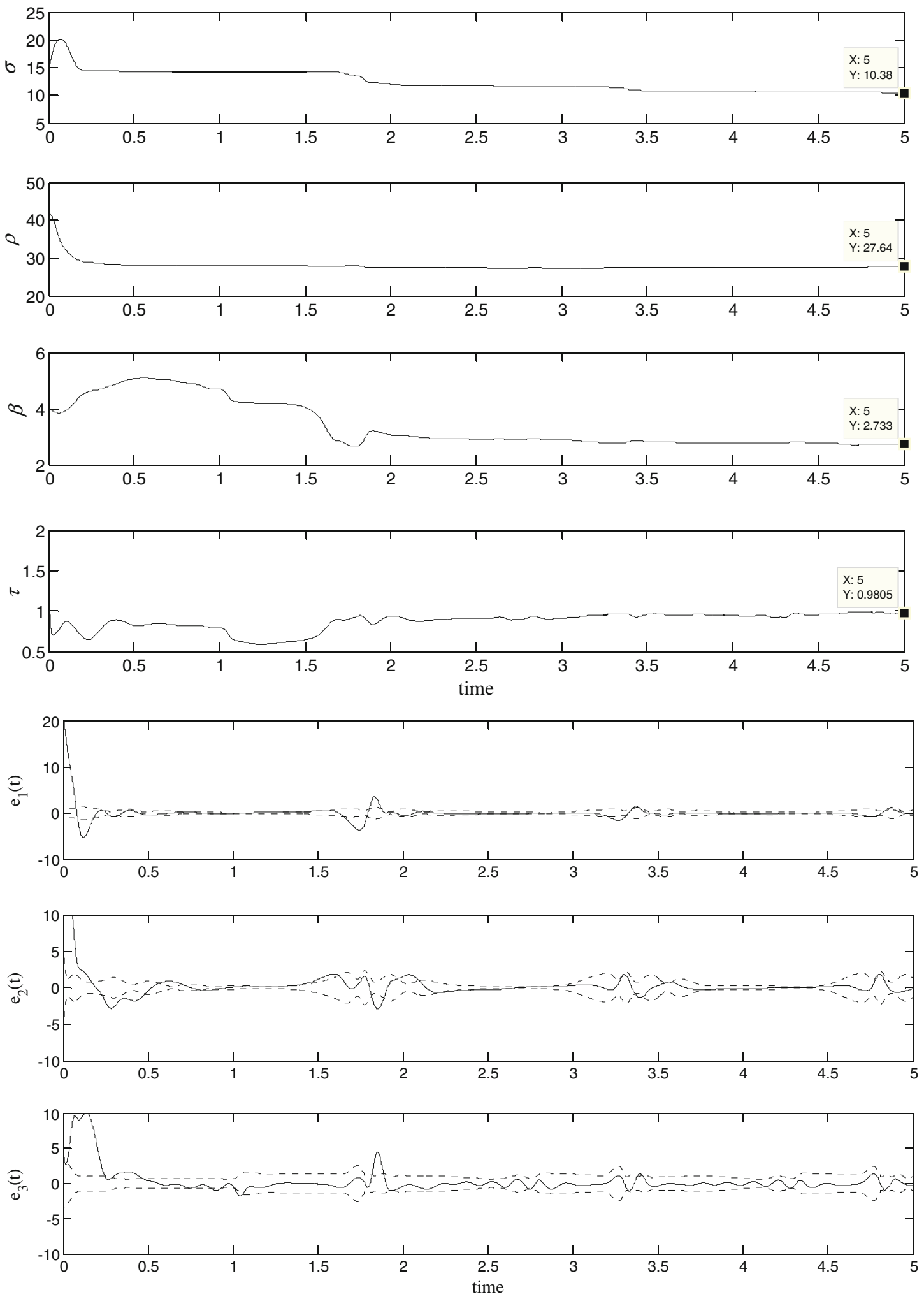

Fig. 12 Estimation of parameters and the delay of the quasi-periodic Lorenz DDE $(\sigma=10, \rho=28, \beta=8 / 3, \tau=1)$ along with the states estimation error (solid line) and $3 \sigma$ error covariance envelope (dotted line) of EKBF 

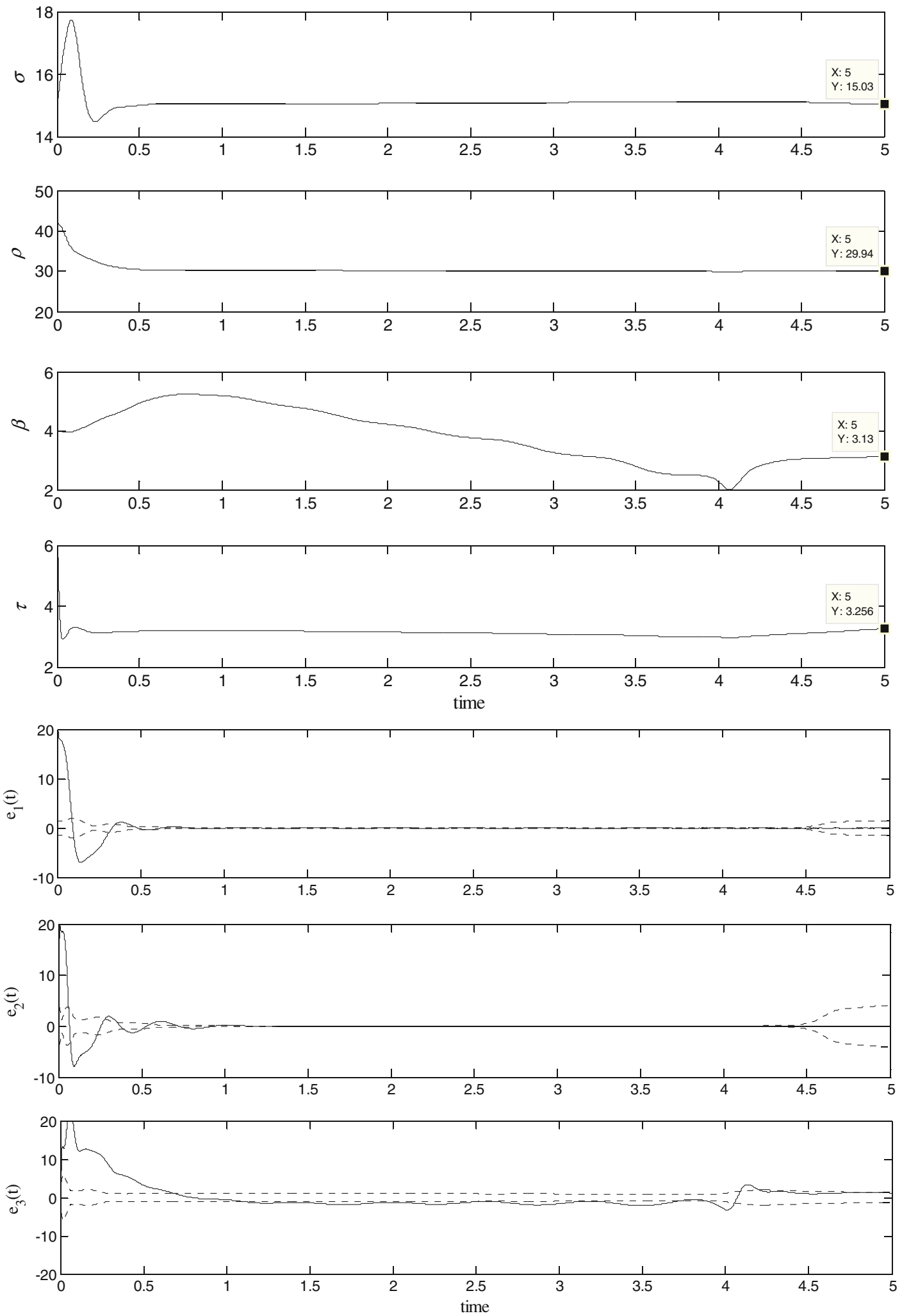

Fig. 13 Estimation of parameters and the delay of the hyperchaotic Lorenz DDE $(\sigma=10, \rho=28, \beta=8 / 3, \tau=4)$ along with the states estimation error (solid line) and $3 \sigma$ error covariance envelope (dotted line) of EKBF 


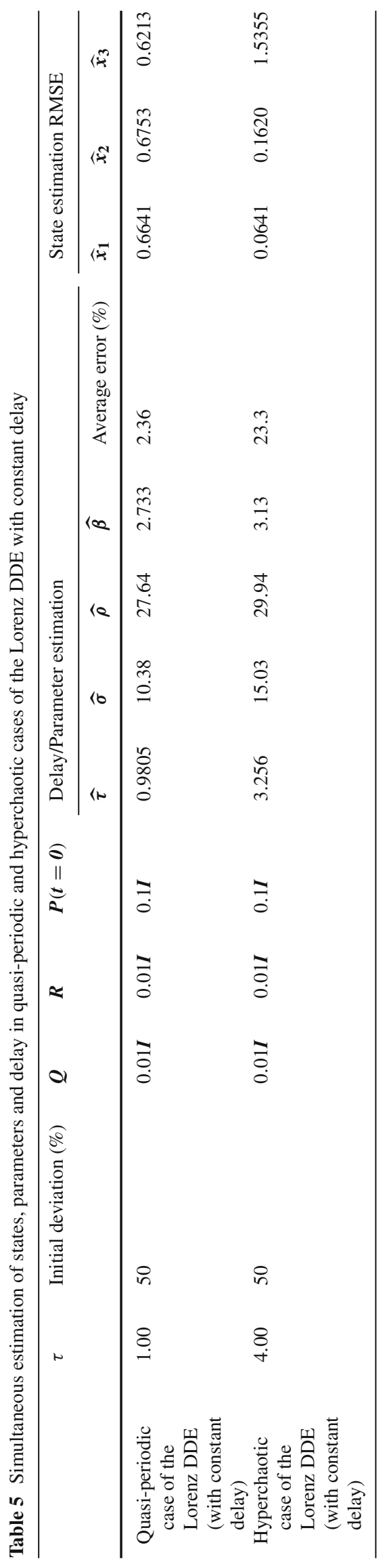



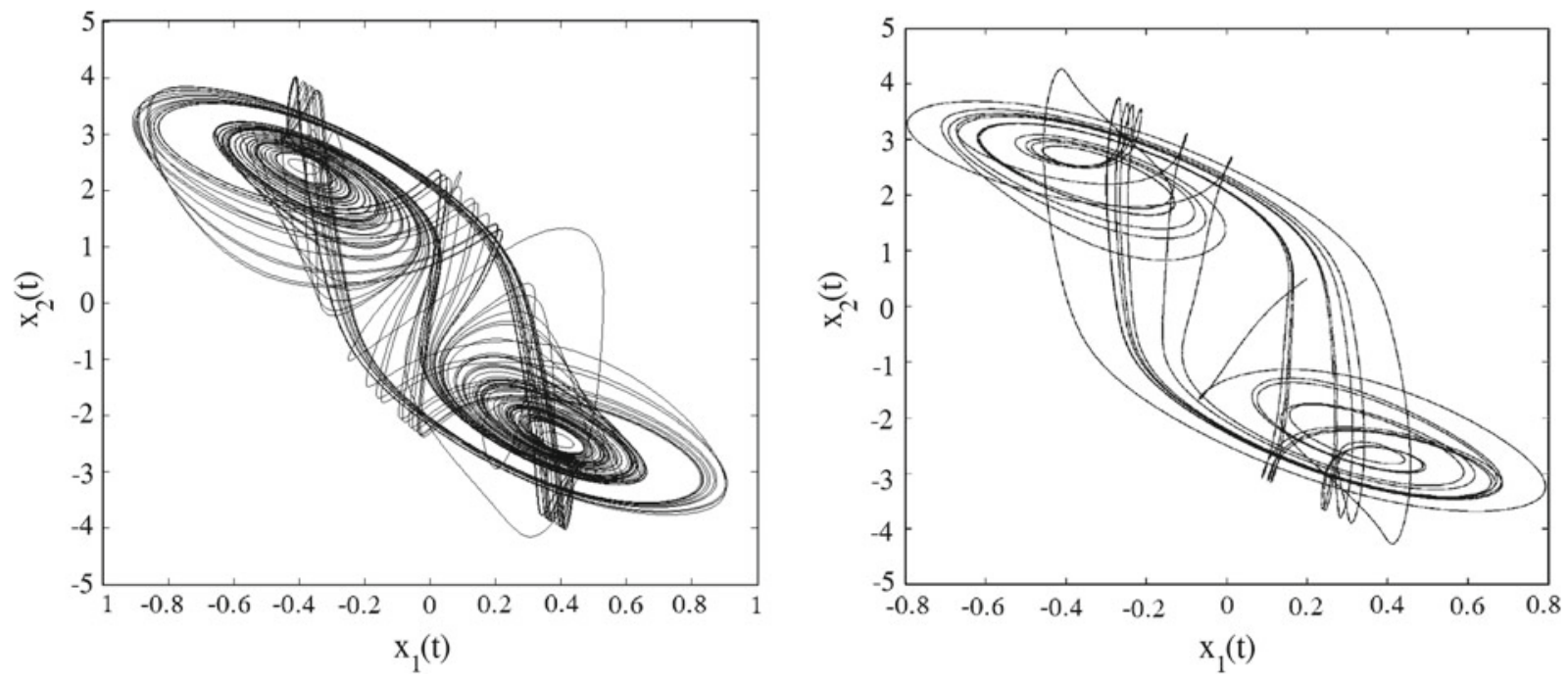

Fig. 14 Phase portrait of the chaotic Hopfield neural network of Eq. (24). With constant delay as in Eq. (25) (left) and time-varying delay as in Eq. (26) (right)

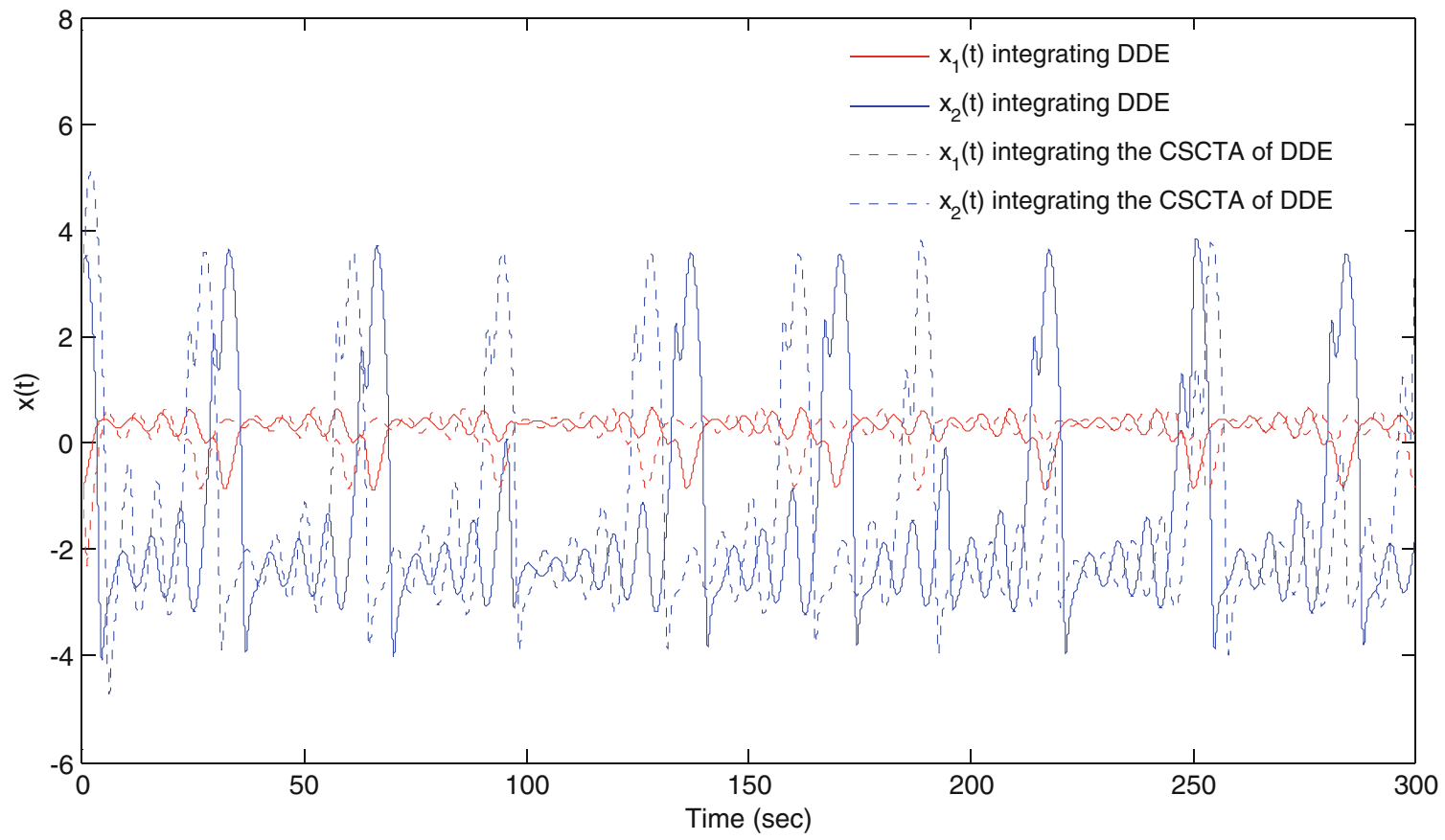

Fig. 15 Chaotic Hopfield neural network of Eq. (24). With constant delay and parameters of Eq. (25) initiated at $\mathrm{x}=[-0.9,3.2]$ obtained from integrating the CSCTA of the DDE compared with those obtained

noise to it. Again the response collected through a measurement function of $\boldsymbol{h}=\mathbf{x}(t)$. A convergence study shows that the time-series of the response within the first $5 \mathrm{~s}$ can be accurately generated through the approximated set of ODEs using 20 collocation points. Therefore, the CSCTA-based model in this case produces reliable predictions and the sole source of uncertainty is the process noise $\mathbf{v}(t)$. Using the CTA, the augmented-state filtering problem can be put in the form of Eq. (10), however, with the time-varying delay of through integrating the original DDE to show the uncertainty in the predictions of the CSCTA-based model

Eq. (22). The EKBF is applied to simultaneously estimate all three states and all three parameters of the system from a noise-corrupted measurement.

The estimation filtering sequence is initiated with a first guess for the augmented state to be $50 \%$ deviated from the true values and using a measurement noise, a process noise and an initial error covariance of $\boldsymbol{R}=\boldsymbol{I}, \boldsymbol{Q}=\boldsymbol{I}$ and $\mathbf{P}_{0}=10 \boldsymbol{I}$, respectively. The estimated states obtained by the EKBF along with the true state and the noise-corrupted measure- 
ments are shown in Fig. 10. Also the estimation errors for all three estimated states along with error covariance envelopes $(3 \sigma)$ are plotted versus time in Fig. 10. The RMSE of the estimated states, along with the estimated values of the unknown parameters after $5 \mathrm{~s}$ are listed in Table 3. As is clear from the results, the approach is capable of accurately estimating the states and all parameters of the chaotic Lorenz DDE with a time-varying delay from a rather uncertain model, a rough initial guess, and a noise-corrupted measurement with only a negligible percentage of error.

\subsubsection{Delay estimation in Lorenz DDE with constant delay}

The proposed estimation technique is applied here for delay and state estimation in the time delayed Lorenz system. Consider Eq. (10) when a constant delay is used i.e. $\tau(t)=\tau$. Two different approaches were described in Sect. 4 for delay estimation. Attempts to use the current estimation technique for delay estimation in this system while using the first approach produce poor results due to the complexity it burdens to the highly nonlinear time delayed system. However, if we take the second approach and change the variable $t$ into $\tilde{t}=\frac{t}{\tau}$ and also write the derivative $\frac{d}{d t}$ in terms of the new variable $\tilde{t}$, then the delay term in the operator $\widetilde{\mathbb{A}}(t)$ will vanish (except for its presence in $\mathbf{A}, \mathbf{B}$ ). Therefore, the delay term in the new format of Eq. (10) will appear as coefficients in $\mathbf{A}, \mathbf{B}$ and $\mathbf{g}$ matrices and it can now be treated as a parameter of the system. Then, a similar procedure as used for simultaneous estimation of states and parameters is applied for simultaneous estimation of states and the delay.

The estimation procedure is applied independently for the chaotic, quasi-periodic and hyperchaotic cases when the true values of the delay are $\tau=\frac{1}{6}, 1$ and 4 . The covariance of the measurement and process noise as well as the initial error covariance used for each case are listed in Table 4. The estimation filtering sequence is initiated with a first guess for the augmented state $50 \%$ deviated from the true values in the chaotic case of $\tau=\frac{1}{6}$ as well as in the hyperchaotic case of $\tau=4$. However, in the quasi-periodic case of $\tau=1$, the initial guess for the augmented state is chosen to be ten times the true value. The time evolution of the estimated delay obtained by extended Kalman-Bucy filter for all three cases of $\tau=\frac{1}{6}, \tau=4$ and $\tau=1$ are shown in Fig. 11a-c, respectively. The RMSE of the estimated states and the estimated values of the unknown delay after $5 \mathrm{~s}$ are listed in Table 4 .

The comparison of the results of this part with those of Sects. 6.2.1 and 6.2.2 shows that the error of the estimated delay for the chaotic and hyperchaotic cases are generally higher than those of the other estimated parameters despite of the lower level of noise used for delay estimation problem. However, the approach seems to be more robust against a large initial error in the delay than it is against a large initial error in the parameters.

\subsubsection{Simultaneous estimation of states, parameters and delay}

In this section the approach is applied to simultaneously estimate all three states of the system, all three parameters of the system, and the delay. Again the change of variable $\tilde{t}=\frac{t}{\tau}$ is used to make the delay term appear as a coefficient of the system. The procedure is implemented for two cases including one quasi-periodic and one hyperchaotic. The true value of the unknown delay for the quasi-periodic case is assumed to be $\tau=1$ and for the hyperchaotic case to be $\tau=4$. The covariance of the measurement and process noise as well as the initial error covariance used for each case are listed in Table 5. The estimated parameters and delay obtained by extended Kalman-Bucy filter for the quasi-periodic cases of $\tau=1$ and the hyperchaotic case of $\tau=4$ along with the estimation errors for the estimated states are shown in Figs. 12 and 13, respectively. The RMSE of the estimated states and the estimated values of the unknown delay and the unknown parameters after $5 \mathrm{~s}$ are listed in Table 5 . The results clearly show that assuming both the delay and the parameters of the system to be unknown significantly reduces the accuracy of the approach. As is expected, the accuracy is reduced more for the hyperchaotic case than for the quasi-periodic case.

\subsection{Hopfield neural network model}

Consider a nonlinear DDE with a single discrete time-varying delay of the form

$$
\begin{aligned}
\dot{\mathbf{x}}(t)= & \mathbf{A} \mathbf{x}(t)+\mathbf{B} \mathbf{x}(t-\tau)+\mathbf{C} \boldsymbol{F}_{1}(t, \mathbf{x}(t)) \\
& +\mathbf{D} \boldsymbol{F}_{2}(t, \mathbf{x}(t-\tau(t)))+\mathbf{I}
\end{aligned}
$$

The nonlinear DDE above is a neural network model known as delayed Hopfield neural network where $\mathbf{x}(t) \in \mathbb{R}^{n}$ is the state vector associated with the neurons, $\mathbf{A}$ is a negative diagonal matrix, $\mathbf{C}$ is the connection weight matrix $\mathbf{D}$ is the delayed connection matrix, and I represents the external input. $\tau(t) \geq 0$ is the inevitable time-delay first realized by Hopfield [44] which occurs in hardware implementation of neural network model due to the finite switching speed of the amplifiers. $\boldsymbol{F}_{1}() \in \mathbb{R}^{n}$ and $\boldsymbol{F} 2() \in \mathbb{R}^{n}$ are the activation functions of the neurons. Here in this study we consider a two-neuron network, i.e. $n=2$ with no external input $(\mathbf{I}=0)$, an activation function $F_{1}(t, \mathbf{x})=F_{2}(t, \mathbf{x})=\tanh (\mathbf{x})$ and $\mathbf{B}=\mathbf{0}$.

Two different cases are considered based on the timedelay being constant or time-varying. For the case of constant time-delay, if we consider the delay $\tau$ and the matrices 


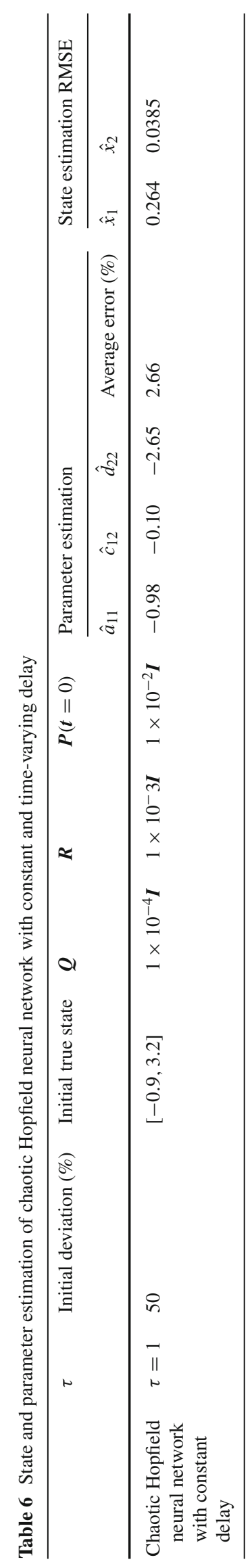



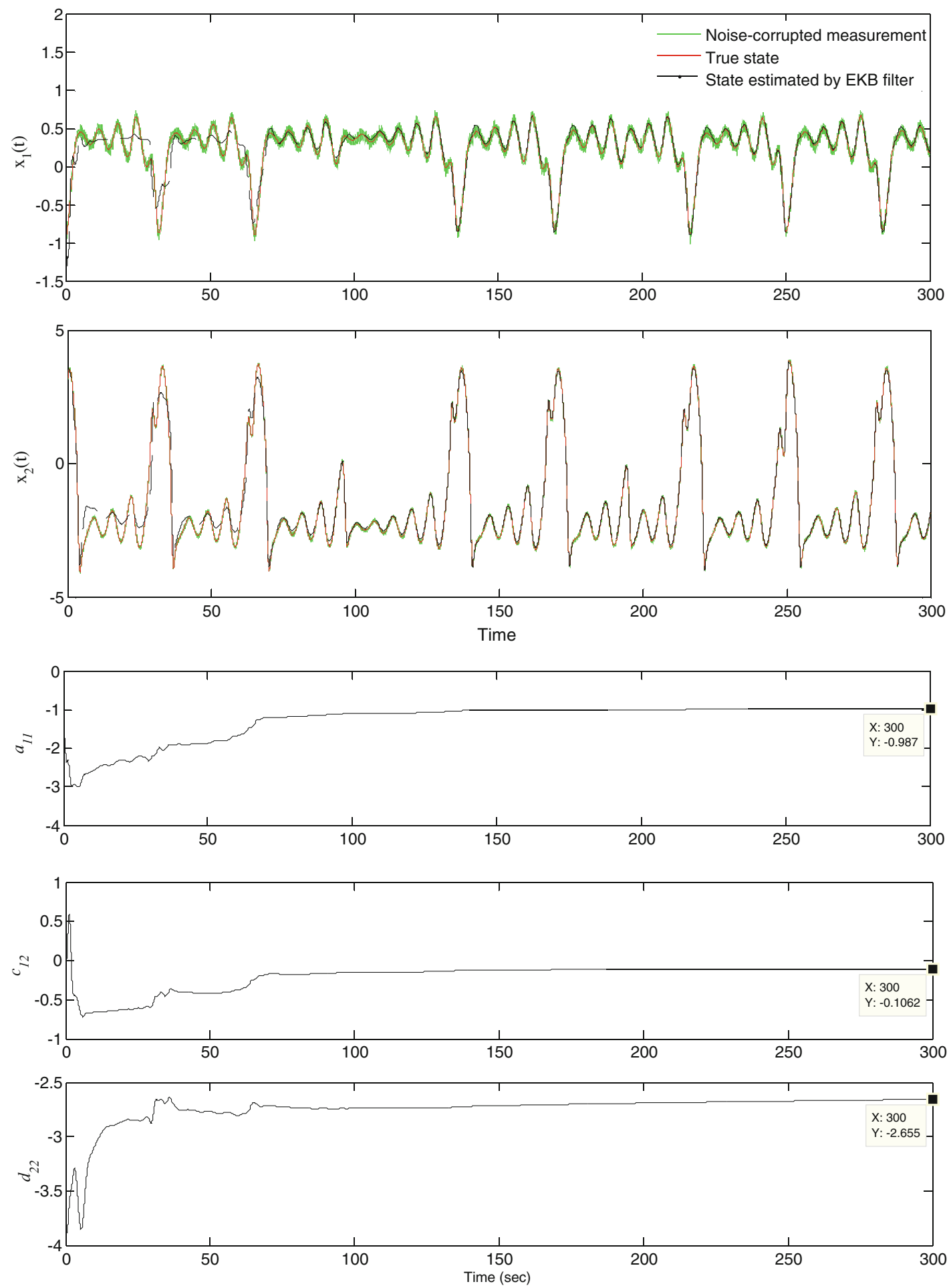

Fig. 16 States of the chaotic Hopfield neural network of Eq. (24). With the delay and parameters of Eq. (25) estimated using EKBF along with the time evolution of the estimated parameters
$\mathbf{A}=a_{i j}, \mathbf{C}=c_{i j}$ and $\mathbf{D}=d_{i j}$ as
$\mathbf{D}=\left[\begin{array}{ll}-1.5 & -0.1 \\ -0.2 & d_{22}\end{array}\right]$
$\tau=1, \quad \mathbf{A}=\left[\begin{array}{ll}-1 & 0 \\ 0 & -1\end{array}\right], \quad \mathbf{C}=\left[\begin{array}{ll}2 & -0.1 \\ -5 & c_{22}\end{array}\right]$
and set $c_{22}$ and $d_{22}$ as bifurcation parameters, by increasing $c_{22}$ from $c_{22}=0.3$ and decreasing $d_{22}$ from $d_{22}=0.2$ 

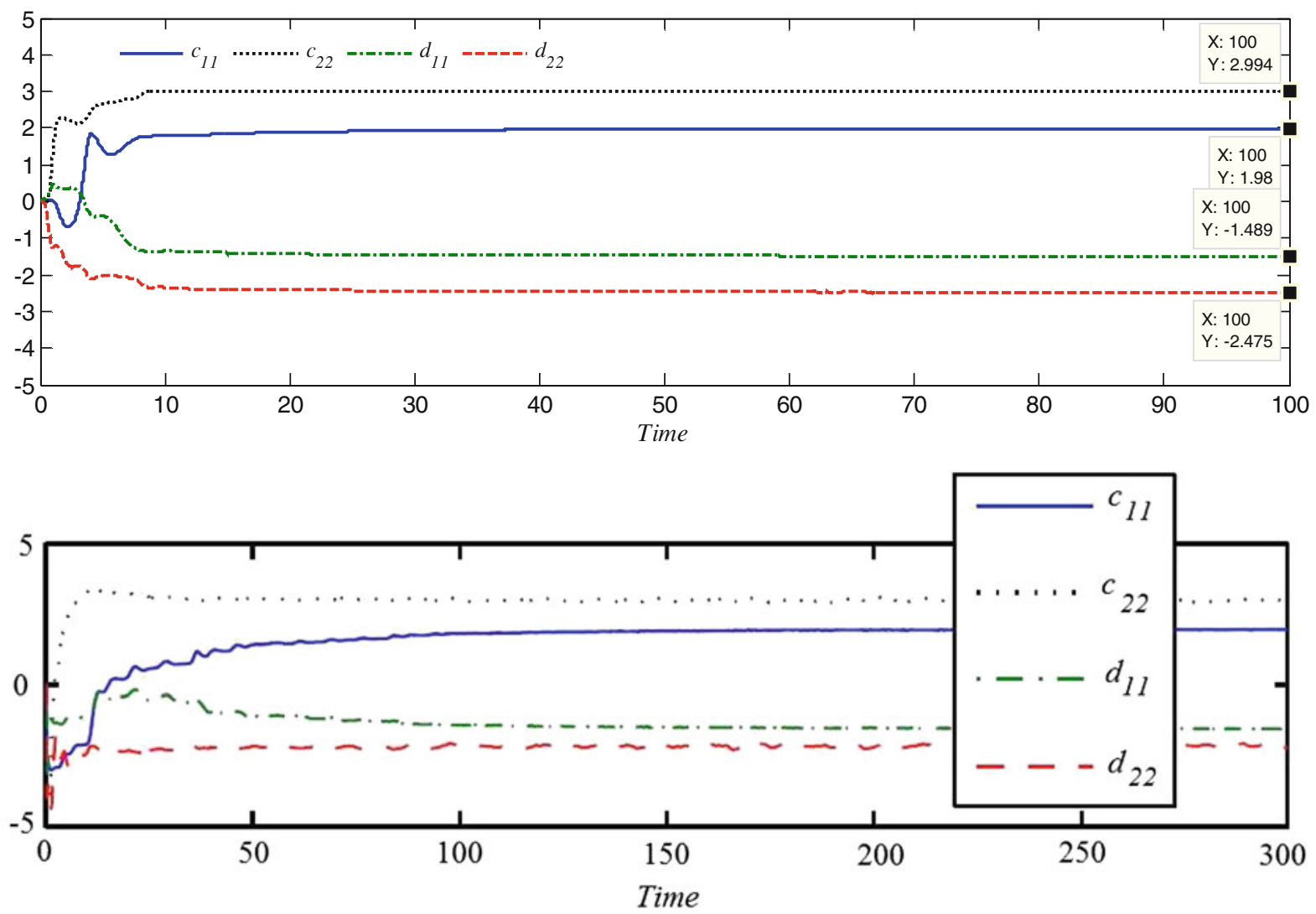

Fig. 17 Estimated parameters of chaotic Hopfield delayed neural network with time varying delay using current approach (top) compared with those in [20] (bottom)

while keeping $c_{22}+d_{22}=0.5$, the system exhibits a perioddoubling route to chaos [45]. Eventually at $c_{22}=3$ and $d_{22}=$ -2.5 , the oscillator exhibits a fully-developed double-scroll chaotic attractor. The phase portrait of the chaotic attractor is depicted in Fig. 14a. For the case of time-varying delay, if the delay $\tau(t)$ and the matrices $\mathbf{A}, \mathbf{C}$ and $\mathbf{D}$ are considered as

$$
\begin{aligned}
& \tau(t)=\frac{e^{t}}{1+e^{t}}, \quad \mathbf{A}=\left[\begin{array}{ll}
-1 & 0 \\
0 & -1
\end{array}\right], \quad \mathbf{C}=\left[\begin{array}{ll}
2 & -0.1 \\
-5 & 3.2
\end{array}\right], \\
& \mathbf{D}=\left[\begin{array}{ll}
-1.6 & -0.1 \\
-0.18 & -2.4
\end{array}\right]
\end{aligned}
$$

The delayed oscillator exhibits chaotic behavior. The phase portrait of the chaotic attractor is depicted in Fig. 14b.

\subsubsection{Chaotic Hopfield neural network with constant delay}

The proposed estimation procedure is applied for the neural network model of Eq. (24) with constant delay and parameters selected as those of Eq. (25) which leads to a chaotic delayed oscillator. A comparison between the response of the Hopfield neural network with $\tau=1$ integrated by the Matlab dde 23 integrator with that obtained from integrating the set of ODEs acquired from CSCTA with 20 collocation points using an 8th order Runge-Kutta integrator is shown in Fig. 15. The comparison clearly shows that owing to the chaotic nature of the delayed oscillator CSCTA gives only a very rough approximation of the solution of the infinite-dimensional oscillator. Despite of the existence of such uncertainty in the process model, the filtering approach is employed to estimate the parameters of the system from a noise-corrupted measurement. The covariance of the measurement and process noise as well as the initial error covariance used for each case are listed in Table 6. The estimation filtering sequence is initiated with a first guess for the augmented state $50 \%$ deviated from the true values. The estimated states and the time evolution of the estimated parameters obtained by extended Kalman-Bucy filter using uncertain CSCTA-based model and noise-corrupted measurement are shown in Fig. 16. The RMSE of the estimated states are also listed in Table 6.

\subsubsection{Chaotic Hopfield neural network with nonlinear time-varying delay}

The proposed estimation procedure is applied for the neural network model of Eq. (24) with the delay and parameters selected as those of Eq. (26) which leads to a chaotic delayed oscillator with time-varying delay. Since parameter estimation for this system with the same parameters and delay 

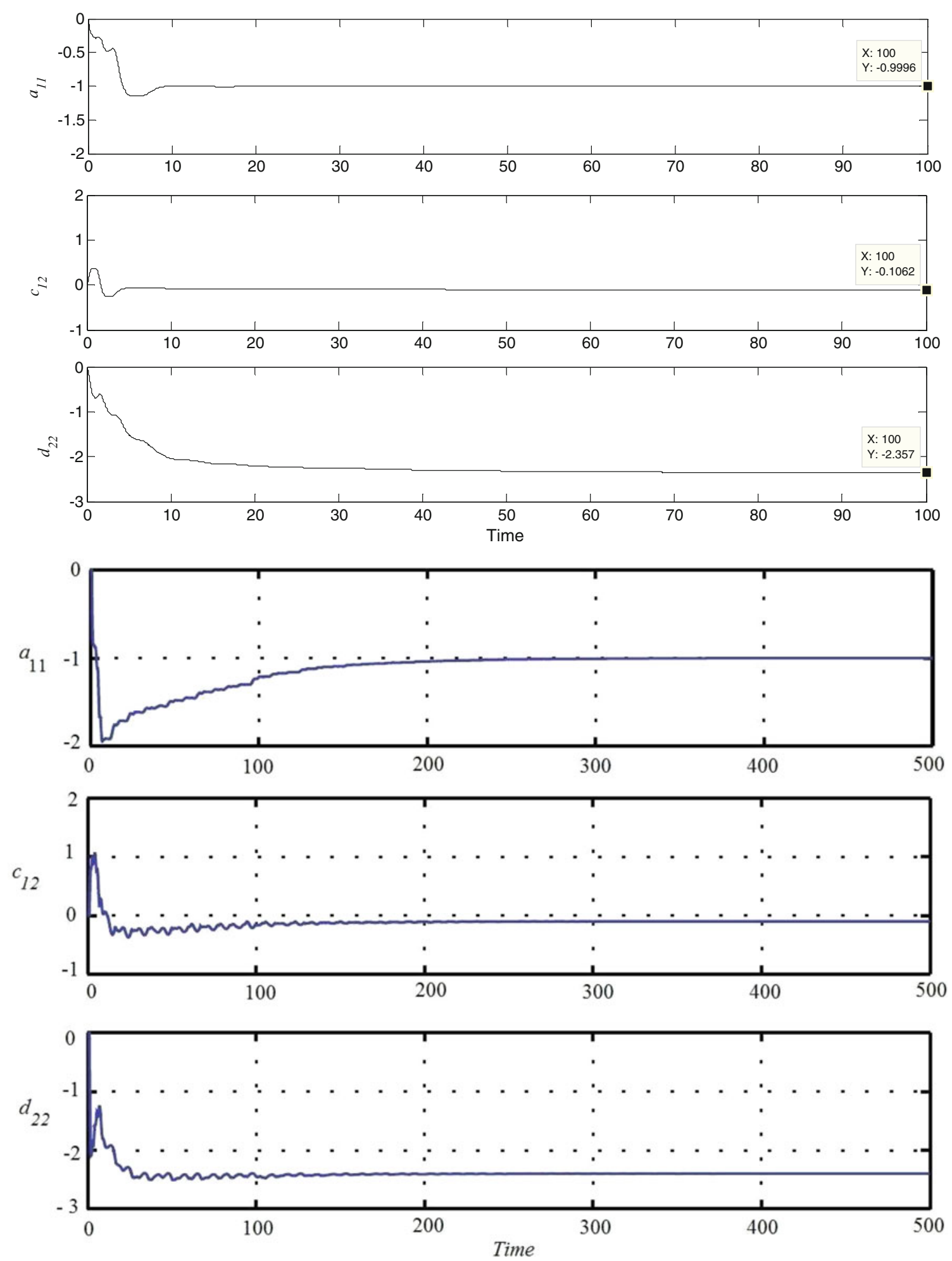

Fig. 18 Estimated parameters of chaotic Hopfield delayed neural network with time varying delay using current approach (up) compared with those of reference [23] (down) 
as those shown in Eq. (26) has previously been studied in $[20,23]$, this system is considered here in order to compare the performance of the current technique with those existing methods. In [20], the unknown parameters to be estimated are $c_{11}, c_{22}, d_{11}$ and $d_{22}$ and measurement noise is added only to the state $x_{1}$ while there is no process noise or any uncertainty present in the model. A combination of synchronization based on dynamical feedback with an adaptive evolution for the unknown parameters is used for parameter estimation in [20]. We here use our proposed approach while the same parameters are assumed to be unknown, however, we consider measurements for both states to be corrupted with noise $\left(\boldsymbol{R}=1 \times 10^{-3} \boldsymbol{I}\right)$ and the model to have both uncertainty (due to the chaotic nature of the system) and process noise $\left(\boldsymbol{Q}=1 \times 10^{-3} \boldsymbol{I}\right)$. The time evolution of the parameters obtained from the current estimation technique is compared with those from [20] in Fig. 17. Since no numerical accuracy of the estimated parameters is given in [20], only a qualitative comparison can be made. As is clear from the figure, despite of the existence of model uncertainty and measurement noise in both states, the current method converges to the true values of parameters within $100 \mathrm{~s}$ while the approach used in [20] takes $300 \mathrm{~s}$ to converge.

In [23], chaos synchronization is used for parameter identification of chaotic delayed system of Eq. (24) with varying time-delay through using an adaptive feedback controller based on the Razumikhin condition and the invariance principle of functional differential equations in the framework of Lyapunov-Krasovskii theory. The unknown parameters in that study are $\mathrm{a}_{11}, \mathrm{c}_{12}$ and $\mathrm{d}_{22}$ and the uncertainties of the model are taken into account but the measurements are assumed to be free of noise. We assume the same parameters to be unknown, however, in our simulation measurements are noise-corrupted $\left(\boldsymbol{R}=1 \times 10^{-3} \boldsymbol{I}\right)$ and the model has uncertainty and process noise $\left(\boldsymbol{Q}=1 \times 10^{-4} \boldsymbol{I}\right)$. The initial conditions of the unknown parameters and those of the states are selected according to [23]. The time evolution of the parameters obtained from the current estimation technique is compared with those from [23] in Fig. 18. Again since no numerical accuracy of the estimated parameters is given in [23], only a qualitative comparison can be made. The results show that despite of the existence of model uncertainty and measurement noise, the current method results in a faster convergence. While the current approach converges to the true values of parameters within $100 \mathrm{~s}$, the approach used in [23] takes $500 \mathrm{~s}$ to converge.

\section{Conclusions}

A novel approach in parameter, delay and state estimation of chaotic and hyperchaotic delayed systems is proposed through exploiting optimal filtering along with CTA. The proposed approach is general and is applicable to the general form of a nonlinear time-varying DDE with time varying delay which includes all well-known delayed systems. The proposed approach is successfully implemented on a variety of forms of the delayed Lorenz system and Hopfield neural network with constant and time-varying delay. The model used in the estimation approach contains two different sources of uncertainties: (1) process noise and (2) the inaccuracy of the CTA which produces large uncertainties in the stats of chaotic and hyperchaotic systems. The extended Kalman-Bucy filter is used to estimate the noise free state as well as the unknown parameters from noise-corrupted measurements. The approach is also shown to be capable of estimating the delay simultaneously with the states of the chaotic system. The approach has been shown to be quite robust against enormous uncertainty of the model, high measurement noise, and large initial errors. One reason for this is due to the fact that, although CSCTA cannot accurately predict the true state of the delayed system at any given instant (unlike the case of non-chaotic linear and nonlinear systems for which CSCTA has been shown to produce accurate state prediction), the chaotic attractor of the finite-dimensional approximation is an accurate projection of the true attractor onto the finite-dimensional state. Also in this paper an innovative technique is proposed to approximately compute the Les of a delayed system through utilizing CSCTA.

The proposed filtering technique is a sequential technique that is capable of estimating all parameters of the system at once in presence of uncertainty in the model. In this approach, unlike the techniques used by other authors [21,22], the effects of uncertainties of the model and external disturbance (noise) of the measurements are taken into account through employing stochastic estimation. Two main advantages of the proposed approach compared with the optimization techniques used by other authors [16,21,22], are:

- Optimization techniques require the entire history of observation while in sequential techniques only the most recent observation in needed and the algorithm can be terminated upon satisfaction of a stopping rule.

- In optimization techniques such as least squares, the dimensions of the matrices increase with time while in sequential filtering the dimensions are fixed throughout the procedure.

Due to the relatively fast convergence of the sequential filtering technique, this approach relies on a limited length of measured data set (as short as $5 \mathrm{~s}$ in some examples studied here).

Acknowledgments Financial support from the National Science Foundation, under the Grant No. CMMI-0900289 is gratefully appreciated. 


\section{References}

1. Mackey MC, Glass L (1977) Oscillation and chaos in physiological control systems. Science 197:287-9

2. Sun J (2004) Global synchronization criteria with channel timedelay for chaotic time-delay systems. Chaos Solitons Fractals 21:967-975

3. Lu H, He Z (1996) Chaotic behavior in first-order autonomous continuous-time systems with delay. IEEE Trans Circuits Syst 43:700-702

4. Sun JT, Zhang YP, Liu YQ, Deng FQ (2002) Exponential stability of interval dynamical system with multidelay. J Appl Math Mech 31(1):95-99

5. Lu H, He Z (1996) Chaotic behavior in first order autonomous continuous time system with delay. IEEE Trans Circuits Syst I 43:700-702

6. Gwynne P (2001) Physicist who makes cash from chaos. Phys World 9:9-9

7. Kocarev L, Parlitz U (1995) General approach for chaotic synchronization with applications to communication. Phys Rev Lett 74:5028

8. Sivaprakasam S, Shore KA (2000) Critical signal strength for effective decoding in diode laser chaotic optical communications. Phys Rev E 61:5997-5999

9. Liu YW, Ge GM, Zhao H, Wang YH, Gao L (2000) Synchronization of hyperchaotic harmonics in time-delay systems and its application to secure communication. Phys Rev E 62:7898

10. Torkamani S, Butcher EA, Todd MD, Park GP (2012) Hyperchaotic probe for damage identification using nonlinear prediction error. Mech Syst Signal Process 29:457-473

11. Torkamani S, Butcher EA, Todd MD, Park GP (2011) Detection of system changes due to damage using a tuned hyperchaotic probe. Smart Mater Struct 20:025006

12. Orlov Y, Belkoura L, Richard JP, Dambrine M (2002) On-line parameter identification of linear time delay systems. In: Proceedings of the 41st IEEE conference on decision and control. Las Vegas, NV, pp 630-635

13. Mann BP, Young KA (2006) An empirical approach for delayed oscillator stability and parametric identification. Proc R Soc A 462:2145-2160

14. Torkamani S, Butcher EA, Khasawneh FA (2012) Parameter identification in periodic delay differential equations with distributed delay. Commun Nonlinear Sci Numer Simul 18(4):1016-1026

15. Basin M, Shi P, Calderon -Alvarez D, (2008) Optimal state filtering and parameter identification for linear time-delay systems. In: Proceedings of the American control conference, Seattle, pp 7-12

16. Deshmukh V (2011) Parametric estimation for delayed nonlinear time-varying dynamical systems. J Comput Nonlinear Dyn 6:041003

17. Torkamani S, Butcher EA (2013) Optimal parameter and state estimation in stochastic time-varying systems with time delay. Commun Nonlinear Sci Numer Simul 18(8):2188-2201

18. Torkamani S, Butcher EA (2013) Stochastic parameter estimation in nonlinear time-delayed vibratory systems with distributed delay. J Sound Vib 332(14):3404-3418

19. Rakshit B, Chowdhury AR, Saha P (2007) Parameter estimation of a delay dynamical system using synchronization in presence of noise. Chaos Solitons Fractals 32:1278-1284

20. Lu JQ, Cao JD (2007) Synchronization-based approach for parameters identification in delayed chaotic neural networks. Physica A 382:672-82

21. Tang Y, Guan X (2009) Parameter estimation for time-delay chaotic systems by particle swarm optimization. Chaos Solitons Fractals 40:1391-1398
22. Tang Y, Guan X (2009) Parameter estimation of chaotic system with time-delay: a differential evolution approach. Chaos Solitons Fractals 42:3132-3139

23. Sun Z, Yang X (2010) Parameters identification and synchronization of chaotic delayed systems containing uncertainties and timevarying delay. Math Prob Eng. doi:10.1155/2010/105309

24. Kosko B (1988) Bidirectional associative memories. IEEE Trans Syst Man Cybern 18(1):49-60

25. Mackey MC, Glass L (1977) Oscillation and chaos in physiological control systems. Science 197(4300):287-289

26. Cao J, Lu J (2006) Adaptive synchronization of neural networks with or without time-varying delay. Chaos 16(1): 013133

27. Sun Z, Xu W, Yang X, Fang T (2006) Inducing or suppressing chaos in a double-well duffing oscillator by time delay feedback. Chaos Solitons Fractals 27(3):705-714

28. Li L, Peng H, Yang Y, Wang X (2009) On the chaotic synchronization of Lorenz systems with time-varying lags. Chaos Solitons Fractals 41:783-794

29. Chua LO, Yang L (1988) Cellular neuaral network: theory. IEEE Trans Circuits Syst 35(10):1257-1272

30. Batkai A, Piazerra S (2005) Semigroups for delay equations. Research notes in mathematics, vol 10. A.K. Peters Ltd., Wellesly

31. Michiels W, Niculescu SI (2008) Stability and stabilization of time-delay systems: an Eigenvalue-based approach. SIAM Press, Philadelphia

32. Sun J-Q (2008) A method of continuous time approximation of delayed dynamical systems. Commun Nonlinear Sci Numer Simul 14(4):998-1007

33. Hale JK, Verduyn Lunel S (1993) Introduction to functional differential equations. Springer, New York

34. Sun J-Q, Song B (2009) Control studies of time-delayed dynamical systems with the method of continuous time approximation. Commun Nonlinear Sci Numer Simul 14:3933-3944

35. Butcher EA, Bobrenkov OA (2011) On the Chebyshev spectral continuous time approximation for constant and periodic delay differential equations. Commun Nonlinear Sci Numer Simul 16:15411554

36. Fox L, Parker IB (1968) Chebyshev polynomials in numerical analysis. Oxford University Press, London

37. Wolf A, Swift J, Swinney H, Vastano J (1985) Determining Lyapunov exponents from a time series. Physica D 16:285

38. Farmer JD (1982) Chaotic attractors of an infinite-dimensional dynamical system. Physica 4D:366-393

39. Ghosh D, Chowdhury R, Saha P (2008) Multiple delay Rossler system: bifurcation and chaos control. Chaos Solitons Fractals $35: 472-485$

40. Jazwinski AH (1970) Stochastic processes and filtering theory. Academic, New York

41. Torkamani S (2013) Hyperchaotic and delayed oscillators for system identification with application to damage assessment. PhD Dissertation, New Mexico State University

42. Benettin G, Galgani L, Giorgilli A, Strelcyn J-M (1980) Lyapunov characteristic exponents for smooth dynamical systems and for Hamiltonian systems: a method for computing all of them. Meccanica $15: 9$

43. Shimada I, Nagashima T (1979) A numerical approach to ergodic problem of dissipative dynamical systems. Prog Theor Phys 61:1605

44. Hopfield JJ (1984) Neurons with graded response have collective computational properties like those of two-state neurons. Proc Natl Acad Sci USA 81:3088-3092

45. Lu H (2002) Chaotic attractors in delayed neural networks. Phys Lett A 298:109-116 